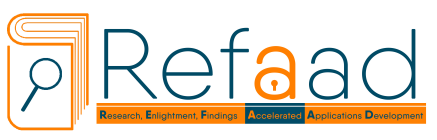

General Letters in Mathematics Vol. 3, No. 1, Aug 2017, pp.1-24

e-ISSN 2519-9277, p-ISSN 2519-9269

Available online at http:// www.refaad.com

\title{
Integrable Systems, Spectral Curves and Representation Theory
}

\author{
A. Lesfari \\ Department of Mathematics, Faculty of Sciences, University of Chouaïb Doukkali, \\ B.P. 20, El Jadida, Morocco. \\ lesfariahmed@yahoo.fr
}

\begin{abstract}
The aim of this paper is to present an overview of the active area via the spectral linearization method for solving integrable systems. New examples of integrable systems, which have been discovered, are based on the so called Lax representation of the equations of motion. Through the Adler-Kostant-Symes construction, however, we can produce Hamiltonian systems on coadjoint orbits in the dual space to a Lie algebra whose equations of motion take the Lax form. We outline an algebraic-geometric interpretation of the flows of these systems, which are shown to describe linear motion on a complex torus. These methods are exemplified by several problems of integrable systems of relevance in mathematical physics.
\end{abstract}

Keywords: Integrable systems, Jacobian varieties, spectral curves.

MSC2010 70H06, 14H40, 14H55.

\section{Introduction}

Last century, mechanics was dominated by the question whether a dynamical system can be solved by quadratures, i.e., by a finite number of algebraic operations including the inverting of functions. This was done, if at all possible, by finding appropriate variables $\left(\alpha_{1}, \ldots, \alpha_{2}\right)$ such that $\left(\alpha_{1}, \ldots, \alpha_{n}, H_{1}, \ldots, H_{n}\right)$ form a local system of canonical coordinates in which the Hamiltonian vector fields $X_{H_{i}}$ take the simple form

$$
X_{H_{j}}: \dot{\alpha}_{i}=\delta_{i j}, \quad \dot{H}_{i}=0 .
$$

Historically, the developments of mechanics and algebraic geometry (in particular the theory of Riemann surfaces) were closely intertwined. This comes from the fact that, in most examples, the quadratures $\alpha_{i}$ were obtained in terms of elliptic or hyperelliptic integrals:

$$
\alpha_{i}=\sum_{k=1}^{g} \int^{s_{k}(t)} \frac{z^{n-i} d z}{\sqrt{P\left(z, c_{j}\right)}},
$$

with $P\left(z, c_{j}\right)$ a polynomial of degree $2 g+1$ or $2 g+2$ in $z$ and the $s_{k}(t)$ some appropriate variables, algebraically related to the originally given ones, for which the Hamilton-Jacobi equation could be solved by separation of variables. The solutions of these problems can be expressed in terms of theta functions related to Riemann surfaces.

The classical approach to proving that a system is integrable by quadratures (in terms of hyperelliptic integrals) was something very unsystematic and required a great deal of luck and ingenuity. Jacobi [24] himself was very much aware of this difficulty and in his famous "Vorlesungen ber Dynamik", in the contex of geodesic flow on the ellipsoid (before introducing the elliptic coordinates), he wrote : "Die Hauptschwierigkeit bei der Integration gegebener Differentialgleichungen scheint in der Einfhrung des richtigen Variablen zu bestehen, zu deren Auffindung es kein allgemeine Regel giebt. Man daher das ungekehrte Verfahren einschlagen und nach erlangter Kenntniss 
einer merkwrdigen Substitution die Probleme aufsuchen, bei welchen dieselbe mit Glck zu brauchen ist" Finally, after Poincaré had recognized that most Hamiltonian systems are not completely integrable, the interest in this subject decreased for more than half a century.

The discovery some years ago by Gardner, Greene, Kruskal and Miura [15] that the Korteweg-de Vries (K-dV) equation $[28]$ :

$$
\frac{\partial u}{\partial t}-6 u \frac{\partial u}{\partial x}+\frac{\partial^{3} u}{\partial x^{3}}=0, \quad u(x, 0)=u(x), x \in \mathbf{R}
$$

could be integrated via inverse spectral methods has generated an enormous number of ideas in the area of Hamiltonian integrable systems. Lax [34] showed that(1) is equivalent to the so-called Lax equation

$$
\dot{A}=[A, B] \equiv A B-B A,
$$

where $=\frac{\partial}{\partial t}$ and $A, B$ are the differential operators in $x: A=\frac{\partial^{2}}{\partial x^{2}}+u, B=\frac{\partial^{3}}{\partial x^{3}}+\frac{3}{2} u \frac{\partial}{\partial x}+u$. The Lax equation means that, under the time evolution of the system, the linear operator $A(t)$ remain similar to $A(0)$. So the spectrum of $A$ is conserved, i.e. it undergoes an isospectral deformation. The eigenvalues of $A$, viewed as functionals, represent the integrals (constants of the motion) of the K-dV equation. Around 1974, Mc Kean and van Moerbeke [43], Dubrovin and Novikov [13] solved the periodic problem for the K-dV equation (for $x \in S^{1}$ ) in terms of a linear motion on a real torus. This torus is the real part of the Jacobi variety of a hyperelliptic curve with branch points defined by the simple periodic and anti-periodic spectrum of $A$. A parallel theory related to these problems had its origin in the periodic Toda lattice (discretized version of the K-dV equation) and Krichever [21] generalized these ideas to differential operators of any order, inspired by specials examples of Zaharov-Shabat, among which is the important Kadomtsev-Petviashvili (KP) equation. Also this theory was generalized to difference operators of any order by van Moerbeke and Mumford [55]. They worked out a systematic method which provides an algebraic map from the invariants manifolds defined by the intersection of the constants of the motion to the Jacobi variety of an algebraic curve associated to Lax equation.

These systems can be realized as straight line motions on a Jacobi variety of a spectral curve. Many problems on Kostant-Kirillov coadjoint orbits in subalgebras of infinite dimensional Lie algebras (Kac-Moody Lie algebras) yield large classes of extended Lax pairs (1). A general statement leading to such situations is given by the AdlerKostant-Symes theorem [1, 29, 53]. This theorem produces Hamiltonian systems having many commuting integrals; some precise results are known for interesting classes of orbits in both the case of finite and infinite dimensional Lie algebras. Paradoxically, the finite-dimensional Lie algebras usually lead to noncompact systems, and the infinitedimensional ones to compact systems. Using the van Moerbeke-Mumford linearization method [55], Adler and van Moerbeke $[2,3]$ showed that the linearized flow could be realized on the jacobian variety $J a c(\mathcal{C})$ (or some sub-abelian variety of it) of the algebraic curve (spectral curve)

$$
\mathcal{C}: P(h, z)=\operatorname{det}(A-z I)=0,
$$

associated to (1). We then construct an algebraic map from the complex invariant manifolds of these hamiltonian systems to the jacobian variety $\operatorname{Jac}(\mathcal{C})$ of the curve $\mathcal{C}$. Therefore all the complex flows generated by the constants of the motion are straight line motions on these jacobian varieties, i.e. the linearizing equations are given by

$$
\sum_{j=1}^{g} \int_{s_{j}(0)}^{s_{j}(t)} \omega_{k}=c_{k} t, 0 \leq k \leq g
$$

where $\omega_{1}, \ldots, \omega_{g}$ span the $g$-dimensional space of holomorphic differentials on the curve $\mathcal{C}$ of genus $g$.

In this paper, I will not discuss the notion of algebraic integrability of Hamiltonian systems. The concept of algebraic integrability varies through the literature. A dynamical system is algebraic completely integrable (in the sense of Adler-van Moerbeke $[5,6,39,56])$ if it can be linearized on a complex algebraic torus $\mathbf{C}^{n} /$ lattice (=Abelian variety). The invariants (often called first integrals or constants) of the motion are polynomials and the phase space coordinates (or some algebraic functions of these) restricted to a complex invariant variety defined by putting these invariants equals to generic constants, are meromorphic functions on an Abelian variety. Moreover, in the coordinates of this Abelian variety, the flows (run with complex time) generated by the constants of the motion are straight lines. However, besides the fact that many hamiltonian completely integrable systems posses this structure, another motivation for its study which sounds more modern is : algebraic completely integrable systems come up systematically whenever you study the isospectral deformation of some linear operator containing a rational 
indeterminate. Indeed the Adler-Kostant-Symes theorem [1, 29, 53] applied to Kac-Moody algebras provides such systems which, by the van Moerbeke-Mumford theorem [55], are algebraic completely integrable. Therefore there are hidden symmetries which have a group theoretical foundation. The concept of algebraic complete integrability is quite effective in small dimensions and has the advantage to lead to global results, unlike the existing criteria for real analytic integrability, which, at this stage are perturbation results. I shall not discuss here the weaker notion of analytic integrability; the perturbation techniques developped in that context are of a totally different nature. In fact, the overwhelming majority of dynamical systems, hamiltonian or not, are non-integrable and possess regimes of chaotic behavior in phase space.

\section{Lax equations and spectral curves}

A Lax equation (with parameter $h$ ) is given by a differential equation of the form

$$
\dot{A}(h, t)=[A(h, t), B(h, t)] \text { or }[B(h, t), A(h, t)], \quad\left(. \equiv \frac{d}{d t}\right)
$$

where

$$
A(h, t)=\sum_{k=l}^{m} A_{k}(t) h^{k}, B(h, t)=\sum_{k=l}^{m} B_{k}(t) h^{k},
$$

are functions depending on a parameter $h$ (spectral parameter) whose coefficients $A_{k}$ and $B_{k}$ are matrices in Lie algebras. The pair $(A, B)$ is called Lax pair. Thereafter, the notation $A(h, t)$ or $A(h)$ or $A(t)$ or simply $A$ will be used indifferently. The bracket [,] is the usual Lie bracket of matrices.

The equation (3) establishes a link between the Lie group theoretical and the algebraic geometric approaches to complete integrability. The solution to (3) has the form $A(t)=g(t) A(0) g(t)^{-1}$, where $g(t)$ is a matrix defined as $\dot{g}(t)=-A(t) g(t)$. We form the polynomial

$$
P(h, z)=\operatorname{det}(A-z I) \text { or } \operatorname{det}(z I-A)
$$

where $z$ is another variable and $I$ the $n \times n$ identity matrix. We define the curve (spectral curve) $\mathcal{C}$, to be the normalization of the complete algebraic curve whose affine equation is

$$
P(h, z)=0 .
$$
(3).

Proof. Let us call $C \equiv A-z I$. Observe that

$$
\dot{P}=\operatorname{det} C \cdot \operatorname{tr}\left(C^{-1} \dot{C}\right)=\operatorname{det} C \cdot \operatorname{tr}\left(C^{-1} B C-B\right)=0,
$$

since $\operatorname{tr} C^{-1} B C=\operatorname{tr} B$. On the other hand

$$
\begin{aligned}
\dot{A}^{n} & =\dot{A} A^{n-1}+A \dot{A} A^{n-2}+\cdots+A^{n-1} \dot{A}, \\
& =[A, B] A^{n-1}+A[A, B] A^{n-2}+\cdots+A^{n-1}[A, B], \\
& =(A B-B A) A^{n-1}+\cdots+A^{n-1}(A B-B A), \\
& =A B A^{n-1}-B A^{n}+\cdots+A^{n} B-A^{n-1} B A \\
& =A\left(B A^{n-1}\right)-\left(B A^{n-1}\right) A+\cdots+A\left(A^{n-1} B\right)-\left(A^{n-1} B\right) A .
\end{aligned}
$$

Since $\operatorname{tr}(X+Y)=\operatorname{tr} X+\operatorname{tr} Y, \operatorname{tr} X Y=\operatorname{tr} Y X, X, Y \in \mathcal{M}_{n}(\mathbf{C})$, we obtain

$$
\frac{d}{d t} \operatorname{tr}\left(A_{h}^{n}\right)=\operatorname{tr} \frac{d}{d t}\left(A_{h}^{n}\right)=0,
$$

and consequently $\operatorname{tr}\left(A^{n}\right)$ are first integrals of motion. 


\section{Completely integrable systems and Kac-Moody Lie algebras}

We have shown that a hamiltonian flow of the type (3) preserves the spectrum of $A$ and therefore its characteristic polynomial. The curve

$$
\mathcal{C}: P(z, h)=\operatorname{det}(A(h)-z I)=0,
$$

is time independent, i.e., its coefficients $\operatorname{tr}\left(A^{n}\right)$ are integrals of the motion (equivalently, $A(t)$ undergoes an isospectral deformation). Some hamiltonian flows on Kostant-Kirillov coadjoint orbits in subalgebras of infinite dimensional Lie algebras (Kac-Moody Lie algebras) yield large classes of extended Lax pairs (3). A general statement leading to such situations is given by the Adler-Kostant-Symes theorem [1, 29, 53] and that we will study below (for more information, one can consult for example [2, 6, 37]).

Theorem 3.1. Let $\mathcal{L}$ be a Lie algebra paired with itself via a nondegenerate, ad-invariant bilinear form $\langle\rangle,, \mathcal{L}$ having a vector space decomposition $\mathcal{L}=\mathcal{K}+\mathcal{N}$ with $\mathcal{K}$ and $\mathcal{N}$ Lie subalgebras. Then, with respect to $\langle$,$\rangle , we have$ the splitting $\mathcal{L}=\mathcal{L}^{*}=\mathcal{K}^{\perp}+\mathcal{N}^{\perp}$ and $\mathcal{K}^{\perp} \approx \mathcal{N}^{*}$ (三 the dual of $\left.\mathcal{N}\right)$ paired with $\mathcal{N}$ via an induced form $\langle\langle\rangle$,$\rangle inherits$ the coadjoint symplectic structure of Kostant and Kirillov; its Poisson bracket between functions $H_{1}$ and $H_{2}$ on $\mathcal{N}^{*}$ reads

$$
\left\{H_{1}, H_{2}\right\}(a)=\left\langle\left\langle a,\left[\nabla_{\mathcal{N}^{*}} H_{1}, \nabla_{\mathcal{N}^{*}} H_{2}\right]\right\rangle\right\rangle, a \in \mathcal{N}^{*} .
$$

Let $V \subset \mathcal{N}^{*}$ be an invariant manifold under the above co-adjoint action of $\mathcal{N}$ on $\mathcal{N}^{*}$ and let $\mathcal{A}(V)$ be the algebra of functions defined on a neighborhood of $V$, invariant under the coadjoint action of $\mathcal{L}$ (which is distinct from the $\mathcal{N}-\mathcal{N}^{*}$ action). Then the functions $H$ in $\mathcal{A}(V)$ lead to commuting Hamiltonian vector fields of the Lax isospectral form

$$
\dot{a}=\left[a, p r_{\mathcal{K}}(\nabla H)\right], p r_{\mathcal{K}} \text { projection onto } \mathcal{K}
$$

Proof. Recall that the gradient $\nabla H$ of a function $H$ on a vector space $E$ is defined by

$$
d H=(\nabla H, d v)_{V}, \quad v \in E, \quad \nabla H \in E^{*},
$$

where $E^{*}$ is the dual of $E$ and $(., .)_{V}$ the pairing between $E, E^{*}$. In the above, $\nabla H \in\left(\mathcal{L}^{*}\right)^{*}=\mathcal{L}$ is the gradient of $H$ when viewed as a function of $\mathcal{L}^{*}(\approx \mathcal{L})$, while we have in general that $p r_{\mathcal{K}}, p r_{\mathcal{N}}, p r_{\mathcal{K}^{\perp}}, p r_{\mathcal{N}^{\perp}}$ are respectively the projections onto $\mathcal{K}, \mathcal{N}, \mathcal{K}^{\perp}, \mathcal{N}^{\perp}$ along $\mathcal{K}^{\perp}, \mathcal{K}^{\perp}, \mathcal{N}^{\perp}, \mathcal{K}^{\perp}$, respectively. Note that if $H \in \mathcal{L}^{*} \approx \mathcal{L}$, then

$$
\nabla_{\mathcal{K}^{\perp}} H=p r_{\mathcal{N}}(\nabla H), \quad \nabla_{\mathcal{N}^{\perp}} H=\operatorname{pr}_{\mathcal{K}}(\nabla H) .
$$

Let $V \subset \mathcal{K}^{\perp}$ be an invariant manifold under the coadjoint action of $\mathcal{N}$ on $\mathcal{K}^{\perp} \approx \mathcal{N}^{*}$. From the identity

$$
\left.\frac{d}{d t} H\left(A d_{g(t)}(a)\right)\right|_{t=0}=0
$$

where

$$
g(t)=1+b t+o(t), \quad b \in \mathcal{L}, \quad a \in V,
$$

we deduce the relation

$$
[\nabla H(a), a]=0, \quad a \in V
$$

or what amounts to the same

$$
\left[a, \nabla_{\mathcal{K}^{\perp}} H\right]=-\left[a, \operatorname{pr}_{\mathcal{K}}(\nabla H)\right]
$$

The Poisson bracket between two functions $H_{1}$ et $H_{2}$ on $\mathcal{N}^{*}$ is written

$$
\left\{H_{1}, H_{2}\right\}(a)=\left\langle\left\langle a,\left[\nabla_{\mathcal{N}^{*}} H_{1}, \nabla_{\mathcal{N}^{*}} H_{2}\right]\right\rangle\right\rangle, a \in \mathcal{N}^{*},
$$

where $\langle\langle.,\rangle$.$\rangle is the natural pairing between \mathcal{N}$ and $\mathcal{N}^{*}$, and where $\nabla_{\mathcal{N}^{*}} H_{1} \in \mathcal{N}$ is the natural gradient of $H$ defined by

$$
d H_{1}(X)=\left\langle\left\langle d X, \nabla_{\mathcal{N}^{*}} H_{1},\right\rangle\right\rangle .
$$

Since $\mathcal{K}^{\perp} \approx \mathcal{N}^{*}$ et $\langle\langle.,\rangle\rangle=.\left.\langle.,\rangle\right|_{.\mathcal{K}^{\perp} \times \mathcal{N}}$, hence

$$
\left\{H_{1}, H_{2}\right\}(a)=\left\langle a,\left[\nabla_{\mathcal{K}^{\perp}} H_{1}, \nabla_{\mathcal{K}^{\perp}} H_{2}\right]\right\rangle .
$$


Suppose now that $H_{1}, H_{2} \in \mathcal{A}(V)$ and satisfying the relation (5). Then, by virtue of the relations (5) and (6) and the fact that $\langle.,$.$\rangle is ad-invariant, we obtain$

$$
\begin{aligned}
\left\{H_{1}, H_{2}\right\} & =\left\langle\left[a, \nabla_{\mathcal{K}^{\perp}} H_{1}\right], \nabla_{\mathcal{K}^{\perp}} H_{2}\right\rangle, \\
& =-\left\langle\left[a, p r_{\mathcal{K}} H_{1}\right], \nabla_{\mathcal{K}^{\perp}} H_{2}\right\rangle, \\
& =-\left\langle a,\left[p r_{\mathcal{K}} H_{1}, \nabla_{\mathcal{K}^{\perp}} H_{2}\right]\right\rangle .
\end{aligned}
$$

Using a similar reasoning for $H_{2}$, we get

$$
\left\{H_{1}, H_{2}\right\}=\left\langle a,\left[p r_{\mathcal{K}} H_{1}, \nabla_{\mathcal{K}^{\perp}} H_{2}\right]\right\rangle .
$$

Since $\mathcal{K}$ is a Lie algebra and $a \in \mathcal{K}^{\perp}$, we obtain $\left\{H_{1}, H_{2}\right\}=0$. The Hamiltonian vector field is written

$$
X_{H_{1}}\left(H_{2}\right)=\left\{H_{1}, H_{2}\right\}=\left\langle\left[\nabla_{\mathcal{K}^{\perp}} H_{1}, a\right], \nabla_{\mathcal{K}^{\perp}} H_{2}\right\rangle
$$

and

$$
X_{H_{1}}(a)=p r_{\mathcal{K} \perp}\left[\nabla_{\mathcal{K}^{\perp}} H_{1}, a\right] .
$$

Consequently, the corresponding hamiltonian flow is

$$
\dot{a}=p r_{\mathcal{K}^{\perp}}\left[\nabla_{\mathcal{K}^{\perp}} H_{1}, a\right], \quad H_{1} \in \mathcal{A}(V),
$$

and from (5), we have

Or $\left[\mathcal{K}^{\perp}, K\right] \subset \mathcal{K}^{\perp}$, and thus

$$
\dot{a}=p r_{\mathcal{K}^{\perp}}\left[a, \nabla_{\mathcal{K}^{\perp}} H_{1}\right] .
$$

$$
\dot{a}=\left[a, \nabla_{\mathcal{K}^{\perp}} H_{1}\right],
$$

which completes the proof.

This theorem produces Hamiltonian systems having many commuting integrals; some precise results are known for interesting classes of orbits in both the case of finite and infinite dimensional Lie algebras. Paradoxically, the finite-dimensional Lie algebras usually lead to noncompact systems, and the infinite-dimensional ones to compact systems.

Any finite dimensional Lie algebra $\mathcal{L}$ with bracket [,] and Killing form $\langle$,$\rangle leads to an infinite dimensional formal$ Laurent series extension

$$
\mathcal{L}=\sum_{-\infty}^{m} A_{i} h^{i}: A_{i} \in \mathcal{L}, m \in \mathbf{Z} \text { free, }
$$

with bracket

$$
\left[\sum A_{i} h^{i}, \sum B_{j} h^{j}\right]=\sum_{i, j}\left[A_{i}, B_{j}\right] h^{i+j}
$$

and ad-invariant, symmetric forms

$$
\left\langle\sum A_{i} h^{i}, \sum B_{j} h^{j}\right\rangle_{k}=\sum_{i+j=-k}\left\langle A_{i}, B_{j}\right\rangle
$$

depending on $k \in \mathbf{Z}$. The forms $\langle,\rangle_{k}$ are non degenerate if $\langle$,$\rangle is so. Let \mathcal{L}_{p, q}(p \leq q)$ be the vector subspace of $\mathcal{L}$, corresponding to powers of $h$ between $p$ and $q$.

A first interesting class of problems is obtained by taking $\mathcal{L}=\mathcal{G l}(n, \mathbf{R})$ and by putting the form $\langle,\rangle_{1}$ on the Kac-Moody extension. Then we have the decomposition into Lie subalgebras

$$
\mathcal{L}=\mathcal{L}_{0, \infty}+\mathcal{L}_{-\infty,-1}=\mathcal{K}+\mathcal{N}
$$

with $\mathcal{K}=\mathcal{K}^{\perp}, \mathcal{N}=\mathcal{N}^{\perp}$ and $\mathcal{K}=\mathcal{N}^{*}$. Consider the invariant manifold $V_{m}, m \geq 1$ in $\mathcal{K}=\mathcal{N}^{*}$, defined as

$$
V_{m}=\left\{A=\sum_{i=1}^{m-1} A_{i} h^{i}+\alpha h^{m}, \alpha=\operatorname{diag}\left(\alpha_{1}, \cdots, \alpha_{n}\right) \text { fixed }\right\},
$$

with $\operatorname{diag}\left(A_{m-1}\right)=0$. From $[2,3]$, we have 
Theorem 3.2. The manifold $V_{m}$ has a natural symplectic structure, the functions

$$
H=\left\langle f\left(A h^{-j}\right), h^{k}\right\rangle_{1},
$$

on $V_{m}$ for good functions $f$ lead to complete integrable commuting hamiltonian systems of the form

$$
\dot{A}=\left[A, p r_{\mathcal{K}}\left(f^{\prime}\left(A h^{-j}\right) h^{k-j}\right)\right], A=\sum_{i=0}^{m-1} A_{i} h^{i}+\alpha h^{m},
$$

and their trajectories are straight line motions on the jacobian of the curve $\mathcal{C}$ of genus $(n-1)(n m-2) / 2$ defined by

$$
P(z, h)=\operatorname{det}(A-z I)=0 .
$$

The coefficients of this polynomial provide the orbit invariants of $V_{m}$ and an independent set of integrals of the motion (of particular interest are the flows where $j=m, k=m+1$ which have the following form

$$
\dot{A}=\left[A, a d_{\beta} a d_{\alpha}^{-1} A_{m-1}+\beta h\right], \beta_{i}=f^{\prime}\left(\alpha_{i}\right),
$$

the flow depends on $f$ through the relation $\beta_{i}=f^{\prime}\left(\alpha_{i}\right)$ only).

Another class is obtained by choosing any semi-simple Lie algebra $L$. Then the Kac-Moody extension $\mathcal{L}$ equipped with the form $\langle\rangle=,\langle,\rangle_{0}$ has the natural level decomposition

$$
\mathcal{L}=\sum_{i \in \mathbf{Z}} L_{i},\left[L_{i}, L_{j}\right] \subset L_{i+j}, \quad\left[L_{0}, L_{0}\right]=0, L_{i}^{*}=L_{-i} .
$$

Let

$$
B^{+}=\sum_{i \geq 0} L_{i}, \quad B^{-}=\sum_{i<0} L_{i}
$$

Then the product Lie algebra $\mathcal{L} \times \mathcal{L}$ has the following bracket and pairing

$$
\begin{aligned}
{\left[\left(l_{1}, l_{2}\right),\left(l_{1}^{\prime}, l_{2}^{\prime}\right)\right] } & =\left(\left[l_{1}, l_{1}^{\prime}\right],-\left[l_{2}, l_{2}^{\prime}\right]\right), \\
\left\langle\left(l_{1}, l_{2}\right),\left(l_{1}^{\prime}, l_{2}^{\prime}\right)\right\rangle & =\left\langle l_{1}, l_{1}^{\prime}\right\rangle-\left\langle l_{2}, l_{2}^{\prime}\right\rangle
\end{aligned}
$$

It admits the decomposition into $\mathcal{K}+\mathcal{N}$ with

$$
\begin{gathered}
\mathcal{K}=\{(l,-l): l \in \mathcal{L}\}, \quad \mathcal{K}^{\perp}=\{(l, l): l \in \mathcal{L}\}, \\
\mathcal{N}=\left\{\left(l_{-}, l_{+}\right): l_{-} \in B^{-}, l_{+} \in B^{+}, p r_{0}\left(l_{-}\right)=p r_{0}\left(l_{+}\right)\right\}, \\
\mathcal{N}^{\perp}=\left\{\left(l_{-}, l_{+}\right): l_{-} \in B^{-}, l_{+} \in B^{+}, p r_{0}\left(l_{+}+l_{-}\right)=0\right\},
\end{gathered}
$$

where $p r_{0}$ denotes projection onto $L_{0}$. Then from the last theorem, the orbits in $\mathcal{N}^{*}=K^{\perp}$ possesses a lot of commuting hamiltonian vector fields of Lax form.

In fact the following result [55] could be thought of as an example of theorem 3.2.

Theorem 3.3. The $N$-invariant manifolds

$$
V_{-j, k}=\sum_{-j \leq i \leq k} L_{i} \subseteq \mathcal{L} \simeq \mathcal{K}^{\perp}
$$

has a natural symplectic structure and the functions $H\left(l_{1}, l_{2}\right)=f\left(l_{1}\right)$ on $V_{-j, k}$ lead to commuting vector fields of the Lax form

$$
i=\left[l,\left(p r^{+}-\frac{1}{2} p r_{0}\right) \nabla H(l)\right], p r^{+} \text {projection onto } B^{+},
$$

their trajectories are straight line motions on the Jacobian of a curve defined by the characteristic polynomial of elements in $V_{-j, k}$ thought of as functions of $h$. (where $\nabla H(l) \in \mathcal{N}$ is the gradient of $H$ thought of as a function on $\mathcal{L})$. 
Using the van Moerbeke-Mumford linearization method, Adler and van Moerbeke [2, 3] showed that the linearized flow could be realized on the jacobian variety $\operatorname{Jac}(\mathcal{C})$ (or some sub-abelian variety of it) of the algebraic curve (spectral curve) $\mathcal{C}$ associated to (3). We then construct an algebraic map from the complex invariant manifolds of these hamiltonian systems to the jacobian variety $\operatorname{Jac}(\mathcal{C})$ of the curve $\mathcal{C}$. Therefore all the complex flows generated by the constants of the motion are straight line motions on these jacobian varieties, i.e. the linearizing equations are given by

$$
\int_{s_{1}(0)}^{s_{1}(t)} \omega_{k}+\int_{s_{2}(0)}^{s_{2}(t)} \omega_{k}+\cdots+\int_{s_{g}(0)}^{s_{g}(t)} \omega_{k}=c_{k} t, 0 \leq k \leq g,
$$

where $\omega_{1}, \ldots, \omega_{g}$ span the $g$-dimensional space of holomorphic differentials on the curve $\mathcal{C}$ of genus $g$. In an unifying approach, Griffiths [18] has found necessary and sufficient conditions on $B$ for the Lax flow (3) to be linearizable on the Jacobi variety of its spectral curve, without reference to Kac-Moody Lie algebras.

\section{Applications}

\subsection{Geodesic flow on $S O(n)$}

In the particular case $m=1$, i.e., for $V_{1}$, we choose

$$
A=X+\alpha h, \quad X \in \operatorname{so}(n) .
$$

In this case, the Hamiltonian flow described by equation (7) (where $\alpha_{i}$ and $\beta_{i}$ can be taken arbitrarily) is reduced to the study of the Euler-Arnold equations for the geodesic flow on $S O(n)$,

$$
\dot{X}=[X, \lambda X], \quad(\lambda X)_{i j}=\lambda_{i j} X_{i j}, \quad \lambda_{i j}=\frac{\beta_{i}-\beta_{j}}{\alpha_{i}-\alpha_{j}},
$$

for a left-invariant diagonal metric $\Sigma \lambda_{i j} X_{i j}$. The natural phase space for this motion is an orbit defined in $S O(n)$ by $[n / 2]$ orbit invariants. By theorem 3.3, the problem is completely integrable and the trajectories are straight lines on $\operatorname{Jac}(\mathcal{C})$ of dimension $(n-2)(n-1) / 2$ and more specifically, on the Prym variety $\operatorname{Prym}\left(\mathcal{C} / \mathcal{C}_{0}\right) \subset J a c(\mathcal{C})$ of dimension $(n(n-1) / 2-[w / 2]) / 2$ induced by the natural involution

$$
\mathcal{C} \longrightarrow \mathcal{C}, \quad(z, h) \longmapsto(-z,-h),
$$

on $\mathcal{C}$ as a result of $X \in \operatorname{so}(n) ; \mathcal{C}_{0}$ is the curve obtained by identifying $(z, h)$ with $(-z,-h)$. This problem is studied in detail for $n=3$ and $n=4$ in several sections through various methods.

\subsection{The problem of the rotation of a solid body about a fixed point}

One of the most fundamental problems of mechanics is the study of the motion of rotation of a solid body around a fixed point. The differential equations of this problem are written in the form

$$
\begin{aligned}
\dot{M} & =M \wedge \Omega+\mu g \Gamma \wedge L, \\
\dot{\Gamma} & =\Gamma \wedge \Omega,
\end{aligned}
$$

where $\wedge$ is the vector product in $\mathbf{R}^{3}, M=\left(m_{1}, m_{2}, m_{3}\right)$ the angular momentum of the solid, $\Omega=\left(\frac{m_{1}}{I_{1}}, \frac{m_{2}}{I_{2}}, \frac{m_{3}}{I_{3}}\right)$ the angular velocity, $I_{1}, I_{2}$ and $I_{3}$, moments of inertia, $\Gamma=\left(\gamma_{1}, \gamma_{2}, \gamma_{3}\right)$ the unitary vertical vector, $\mu$ the mass of the solid, $g$ the acceleration of gravity, and finally, $L=\left(l_{1}, l_{2}, l_{3}\right)$ the unit vector originating from the fixed point and directed towards the center of gravity; all these vectors are considered in a mobile system whose coordinates are fixed to the main axes of inertia. The configuration space of a solid with a fixed point is the group of rotations $S O(3)$. This is generated by the one-parameter subgroup of rotations

$$
\begin{gathered}
A_{1}=\left(\begin{array}{ccc}
1 & 0 & 0 \\
0 & \cos t & -\sin t \\
0 & \sin t & \cos t
\end{array}\right), \quad A_{2}=\left(\begin{array}{ccc}
\cos t & 0 & \sin t \\
0 & 1 & 0 \\
-\sin t & 0 & \cos t
\end{array}\right), \\
A_{3}=\left(\begin{array}{ccc}
\cos t & -\sin t & 0 \\
\sin t & \cos t & 0 \\
0 & 0 & 1
\end{array}\right) .
\end{gathered}
$$


Recall that this is the group of $n \times n$ orthogonal matrices $A$ and the motion of this solid is described by a curve on this group. The angular velocity space of all rotations (the set of derivatives $\left.\dot{A}(t)\right|_{t=0}$ of the differentiable curves in $S O(3)$ passing through the identity in $t=0: A(0)=I)$ is the Lie algebra of the group $S O(3)$; it is the algebra $s o(3)$ of the $3 \times 3$ antisymmetric matrices. This algebra is generated as a vector space by the matrices

$$
\begin{gathered}
e_{1}=\left.\dot{A}_{1}(t)\right|_{t=0}=\left(\begin{array}{ccc}
0 & 0 & 0 \\
0 & 0 & -1 \\
0 & 1 & 0
\end{array}\right), \quad e_{2}=\left.\dot{A}_{2}(t)\right|_{t=0}=\left(\begin{array}{ccc}
0 & 0 & 1 \\
0 & 0 & 0 \\
-1 & 0 & 0
\end{array}\right), \\
e_{3}=\left.\dot{A}_{3}(t)\right|_{t=0}=\left(\begin{array}{ccc}
0 & -1 & 0 \\
1 & 0 & 0 \\
0 & 0 & 0
\end{array}\right),
\end{gathered}
$$

which verify the commutation relations :

$$
\left[e_{1}, e_{2}\right]=e_{3} \quad\left[e_{2}, e_{3}\right]=e_{1}, \quad\left[e_{3}, e_{1}\right]=e_{2}
$$

We will use the fact that if we identify $s o(3)$ to $\mathbf{R}^{3}$ by sending $\left(e_{1}, e_{2}, e_{3}\right)$ on the canonical basis of $\mathbf{R}^{3}$, the bracket of $s o(3)$ corresponds to the vector product. In other words, consider the application

$$
\mathbf{R}^{3} \longrightarrow s o(3), a=\left(a_{1}, a_{2}, a_{3}\right) \longmapsto A=\left(\begin{array}{ccc}
0 & -a_{3} & a_{2} \\
a_{3} & 0 & -a_{1} \\
-a_{2} & a_{1} & 0
\end{array}\right),
$$

which defines an isomorphism between Lie algebras $\left(\mathbf{R}^{3}, \wedge\right)$ et $(s o(3),[]$,$) where$

$$
a \wedge b \longmapsto[A, B]=A B-B A .
$$

By using this isomorphism, the system (8) can be rewritten in the form

$$
\begin{aligned}
\dot{M} & =[M, \Omega]+\mu g[\Gamma, L], \\
\dot{\Gamma} & =[\Gamma, \Omega],
\end{aligned}
$$

where

$$
\begin{aligned}
& M=\left(M_{i j}\right)_{1 \leq i, j \leq 3} \equiv \sum_{i=1}^{3} m_{i} e_{i} \equiv\left(\begin{array}{ccc}
0 & -m_{3} & m_{2} \\
m_{3} & 0 & -m_{1} \\
-m_{2} & m_{1} & 0
\end{array}\right) \in s o(3), \\
& \Omega=\left(\Omega_{i j}\right)_{1 \leq i, j \leq 3} \equiv \sum_{i=1}^{3} \omega_{i} e_{i} \equiv\left(\begin{array}{ccc}
0 & -\omega_{3} & \omega_{2} \\
\omega_{3} & 0 & -\omega_{1} \\
-\omega_{2} & \omega_{1} & 0
\end{array}\right) \in \operatorname{co}(3), \\
& \Gamma=\left(\gamma_{i j}\right)_{1 \leq i, j \leq 3} \equiv \sum_{i=1}^{3} \gamma_{i} e_{i} \equiv\left(\begin{array}{ccc}
0 & -\gamma_{3} & \gamma_{2} \\
\gamma_{3} & 0 & -\gamma_{1} \\
-\gamma_{2} & \gamma_{1} & 0
\end{array}\right) \in s o(3),
\end{aligned}
$$

and

$$
L=\left(\begin{array}{ccc}
0 & -l_{3} & l_{2} \\
l_{3} & 0 & -l_{1} \\
-l_{2} & l_{1} & 0
\end{array}\right) \in s o(3)
$$

Taking into account that $M=I \Omega$, then the above equations become

$$
\begin{aligned}
\dot{M} & =[M, \Lambda M]+\mu g[\Gamma, L], \\
\dot{\Gamma} & =[\Gamma, \Lambda M],
\end{aligned}
$$

where

$$
\Lambda M=\equiv \sum_{i=1}^{3} \lambda_{i} m_{i} e_{i} \equiv\left(\begin{array}{ccc}
0 & -\lambda_{3} m_{3} & \lambda_{2} m_{2} \\
\lambda_{3} m_{3} & 0 & -\lambda_{1} m_{1} \\
-\lambda_{2} m_{2} & \lambda_{1} m_{1} & 0
\end{array}\right) \in s o(3), \quad \lambda_{i} \equiv \frac{1}{I_{i}}
$$


The solution of this problem was analyzed first by Euler [14] and in 1758 he published the equations (case $\mu=0$ ) which carry his name. Euler's equations were integrated by Jacobi [24] in terms of elliptic functions and around 1851, Poinsot [51] gave them a remarkable geometric interpretation. Before, around 1815 Lagrange [33] found another case $\left(I_{1}=I_{2}, l_{1}=l_{2}=0\right)$ of integrability, that subsequently Poisson lengthily examined. The problem continued to attract mathematicians but for a long time no new results could be obtained. It was then around 1888-1989 that a memoir [31], of the highest interest, appears containing a new case $\left(I_{1}=I_{2}=2 I_{3}, l_{3}=0\right)$ of integrability discovered by Kowalewski. For this remarkable work, Kowalewski was awarded the Bordin Prize of the Paris Academy of Sciences. In fact, although Kowalewski's work is quite important, it is not at all clear why there would be no other new cases of integrability. This was to be the starting point of a series of fierce research on the question of the existence of new cases of integrability. Moreover, among the remarkable results obtained by Poincaré [50] with the aid of the periodic solutions of the equations of dynamics, we find the following (around 1891): in order to exist in the motion of a solid body around of a fixed point, an algebraic first integral not being reduced to a combination of the classical integrals, it is necessary that the ellipsoid of inertia relative to the point of suspension is of revolution. In 1896, R. Liouville (not to be confused with Joseph Liouville, well known in complex analysis) also competed for the Bordin prize, presented a paper [41] indicating necessary and sufficient conditions $\left(I_{3}=0,2 I_{3} / I_{1}=\right.$ integer $)$ of existence of a fourth algebraic integral. These conditions have been reproduced in most conventional treatises (eg Whittaker [57]) and in scientific journals. And it was not until the year 1906, when Husson [23], working under the direction of Appell and Painlevé, discovered an erroneous demonstration in the work of Liouville. Indeed, paragraphs I and III of Liouville's dissertation devoted to the search for the necessary conditions seem at first satisfactory, but a more careful study shows that the demonstrations are at least insufficient and that it is impossible to accept conclusions. In fact, although the conditions found by Liouville are necessary, they can not be deduced from the calculations indicated and these conditions are not sufficient. And it was Husson who first solved completely the question of looking for new cases of integrability. Inspired by Poincaré's research on the problem of the three bodies and Painlevé [48] on the generalization of Bruns's theorem, Husson demonstrated that any algebraic integral is a combination of classical integrals except in the cases of Euler, Lagrange and Kowalewski. Moreover, the question of the existence of analytic integrals has been studied rigorously by Ziglin [58, 59] and Holmes-Marsden [22]. some special cases could be found : cases of Hesse-Appel'rot [21, 7], Goryachev-Chaplygin [16, 11] and Bobylev-Steklov.

The Euler rigid body motion : In this case we have $l_{1}=l_{2}=l_{3}=0$, that is, the fixed point is its center of gravity. The Euler rigid body motion (also called Euler-Poinsot motion of the solid) express the free motion of a rigid body around a fixed point. Then the motion of the body is governed by

$$
\dot{M}=[M, \Lambda M], \text { or } \dot{M}=[M, \Omega]
$$

Equation (11) admits two first integrals

$$
\left.H_{1}=\frac{1}{2}\left(\lambda_{1} m_{1}^{2}+\lambda_{2} m_{2}^{2}+\lambda_{3} m_{3}^{2}\right), \quad \text { (Hamiltonian }\right)
$$

and

$$
H_{2}=\frac{1}{2}\left(m_{1}^{2}+m_{2}^{2}+m_{3}^{2}\right) .
$$

We shall use the Lax representation of the equations of motion to show that the linearized Euler flow can be realized on an elliptic curve. The solution to the equation (11), has the form

$$
M(t)=O(t) M(t) M^{\top}(t),
$$

where $O(t)$ is one parameter sub-group of $S O(3)$. So the hamiltonian flow (11) preserves the spectrum of $M$ and therefore its characteristic polynomial

$$
\operatorname{det}(M-z I)=-z\left(z^{2}+m_{1}^{2}+m_{2}^{2}+m_{3}^{2}\right) .
$$

Unfortunately, the spectrum of a $3 \times 3$ skew-symmetric matrix provides only one piece of information; the conservation of energy does not appear as part of the spectral information. Therefore one is let to considering another formulation. The basic observation, due to Manakov [42], is that equation (11) is equivalent to the Lax equation

$$
\dot{A}=[A, B],
$$

where

$$
A=M+\alpha h, \quad B=\Lambda M+\beta h,
$$


with a formal indeterminate $h$ and

$$
\begin{gathered}
\alpha=\left(\begin{array}{ccc}
\alpha_{1} & 0 & 0 \\
0 & \alpha_{2} & 0 \\
0 & 0 & \alpha_{3}
\end{array}\right), \quad \beta=\left(\begin{array}{ccc}
\alpha_{1} & 0 & 0 \\
0 & \beta_{2} & 0 \\
0 & 0 & \beta_{3}
\end{array}\right), \\
\lambda_{1}=\frac{\beta_{3}-\beta_{2}}{\alpha_{3}-\alpha_{2}}, \quad \lambda_{2}=\frac{\beta_{1}-\beta_{3}}{\alpha_{1}-\alpha_{3}}, \quad \lambda_{3}=\frac{\beta_{2}-\beta_{1}}{\alpha_{2}-\alpha_{1}},
\end{gathered}
$$

and all $\alpha_{i}$ distinct. The characteristic polynomial of $A$ is

$$
\begin{aligned}
P(h, z) & =\operatorname{det}(A-z I), \\
& =\operatorname{det}(M+\alpha h-z I) \\
& =\prod_{j=1}^{3}\left(\alpha_{j} h-z\right)+\left(\sum_{j=1}^{3} \alpha_{j} m_{j}^{2}\right) h-\left(\sum_{j=1}^{3} m_{j}^{2}\right) z .
\end{aligned}
$$

The spectrum of the matrix $A=M+\alpha h$ as a function of $h \in \mathbf{C}$ is time independent and is given by the zeroes of the polynomial $P(h, z)$, thus defining an algebraic curve (spectral curve). Letting $w=h / z$, we obtain the following elliptic curve

$$
z^{2} \prod_{j=1}^{3}\left(\alpha_{j} w-1\right)+2 H_{1} w-2 H_{2}=0 .
$$

Finally, we have the

Theorem 4.1. The Euler rigid body motion is a completely integrable system and the linearized flow can be realized on an elliptic curve.

The Lagrange top : In this case, we have $I_{1}=I_{2}, l_{1}=l_{2}=0$, i.e., the Lagrange top is a rigid body, in which two moments of inertia are the same and the center of gravity lies on the symmetry axis. In other words, the Lagrange top is a symmetric top with a constant vertical gravitational force acting on its centre of mass and leaving the base point of its body symmetry axis fixed. As in the case of Euler, we show that in this case also the problem is solved by elliptic integrals. Or what amounts to the same, the integration is done using elliptic functions. The Lagrange top is another example of $V_{2}$ in theorem 3.3, we consider the Lagrange top which evolves on an orbit of type $V_{2} ; n=3$,

$$
A=\Gamma+M h+c h^{2} .
$$

Recall that $\Gamma \in s o(3) \simeq \mathbf{R}^{3}$ is the unit vector in the direction of gravity and $M \in s o(3) \simeq \mathbf{R}^{3}$ is the angular momentum in body coordinates with regard to the fixed point; moreover $c=(\lambda+\mu) \Upsilon$ where $\Upsilon \in s o(3) \simeq \mathbf{R}^{3}$ expresses the coordinates of the center of mass and where $(\lambda+\mu, \lambda+\mu, 2 \lambda)$ is the inertia tensor in diagonalized form. The situation then leads to a linear flow on an elliptic curve.

The Kowalewski top : This top is special symmetric top with a unique ratio of the moments of inertia satisfy the relation : $I_{1}=I_{2}=2 I_{3}, l_{3}=0$; in which two moments of inertia are equal, the third is half as large, and the center of gravity is located in the plane perpendicular to the symmetry axis (parallel to the plane of the two equal points). Moreover, we may choose $l_{2}=0, \mu g l_{1}=l$ and $I_{3}=1$. The system in question admits four first integrals :

$$
\begin{aligned}
& H_{1} \equiv H \\
& H_{2}=m_{1} \gamma_{1}+m_{2} \gamma_{2}+m_{3} \gamma_{3} \\
& H_{3}=\gamma_{1}^{2}+\gamma_{2}^{2}+\gamma_{3}^{2} \\
& H_{4}=\left(\left(\frac{m_{1}+i m_{2}}{2}\right)^{2}-\left(\gamma_{1}+i \gamma_{2}\right)\right)\left(\left(\frac{m_{1}-i m_{2}}{2}\right)^{2}-\left(\gamma_{1}-i \gamma_{2}\right)\right) .
\end{aligned}
$$

The fourth first integral was obtained by Kowalewski [31]. During several years and despite many attempts concerning there was no known Lax form for the Kowalewski spinning top for its resolution using the isospectral deformation method. Subsequently, various forms of Lax have been proposed. A detailed geometrical study of Kowalewski's case has revealed rational relationships between various systems. For example, for a long time no one had suspected a 
rational relationship between the Kowalewski spinning top, the geodesic flow over the $S O(4)$ group for the Manakov metric and the Hnon-Heiles differential system. Such a relation was obtained by Adler-van Moerbeke [4] and the use of our results obtained in [26] allowed them to provide a pair of Lax for the case of Kowalewski , Which had been open for several years. Similarly, Haine-Horozov [20] show that on each level surface of the invariants, the equations of the Kowalewski top are equivalent to a Neumann system describing the motion of a mass point on the sphere. This allows us to write a global Lax pair for the Kowalewski system and to show that Kowalewski's original reduction of the integration of the equations of motion to Jacobi's inversion theorem is identical to the introduction of elliptical spherical coordinates for integrating C. Neumann's system. In what follows, we will use the form of Lax (see [6] and references cited in bibliography) in order to linearize the Kowalewski problem via the spectral curve method.

Theorem 4.2. The Kowalewski system admits the following Lax pair

$$
\dot{A}=i[A, B],
$$

with

$$
\begin{gathered}
A=\left(\begin{array}{cccc}
0 & -\Gamma_{2} & -\frac{1}{2} x_{2} h & -\gamma_{3} \\
\Gamma_{1} & 0 & \gamma_{3} & \frac{1}{2} x_{1} h \\
-\frac{1}{2} x_{1} h & -\gamma_{3} & -m_{3} h & \Gamma_{1}-h^{2} \\
\gamma_{3} & \frac{1}{2} x_{2} h & -\Gamma_{2}+h^{2} & m_{3} h
\end{array}\right), \\
B=\left(\begin{array}{cccc}
-m_{3} & 0 & \frac{1}{2} x_{2} & 0 \\
0 & m_{3} & 0 & -\frac{1}{2} x_{1} \\
\frac{1}{2} x_{1} & 0 & m_{3} & h \\
0 & -\frac{1}{2} x_{2} & -h & m_{3}
\end{array}\right)
\end{gathered}
$$

where $x_{1}-m_{1}+i m_{2}, x_{2}=m_{1}-i m_{2}, \Gamma_{1}=\gamma_{1}+i \gamma_{2}, \Gamma_{2}=\gamma_{1}-i \gamma_{2}$.

Proof. We sketch a proof. The projective complex algebraic curve (or spectral curve), defined by the affine equation

is written explicitly

$$
\mathcal{C}: P(h, z)=\operatorname{det}(A-z I)=0,
$$

$$
z^{4}+\left(h^{4}-c_{1} h^{2}+2 c_{3}\right) z^{2}+c_{4} h^{4}+\left(c_{2}^{2}-c-1 c-3\right) h^{2}+c_{3}^{2}=0
$$

where the constants (supposed generic) $c_{1}, c_{2}, c_{3}, c_{4}$ denote respectively the constants $H_{1}, H_{2}, H_{3}, H_{4}(12)$ and this equation describes isospectral deformation. The curve $\mathcal{C}$ is smooth and is a double cover

$$
\varphi: \mathcal{C} \longrightarrow \mathcal{H}, \quad(z, h) \longmapsto(w, h), \quad w=\frac{2 z^{2}+h^{4}-c_{1} h^{2}+2 c_{3}}{h},
$$

of a hyperelliptic curve $\mathcal{H}$ defined by

$$
\mathcal{H}: w^{2}=\left(\left(h^{2}-c_{1}\right)^{2}+4\left(c_{3}-c_{4}\right)\right) h^{2}-4 c_{2}^{2}
$$

The projection $\pi: \mathcal{H} \longrightarrow \mathbf{C},(w, h) \longmapsto h$, realizes $\mathcal{H}$ as a double cover of $\mathbf{C}$ branched in six points. Consequently, the compactified $\overline{\mathcal{H}}$ of $\mathcal{H}$ is a hyperelliptic curve of genus 2. The covering $\varphi$ has four branch points $(w, h)$ on $\mathcal{H}$ for which $z=0$, i.e., $w h=h^{4}-c_{1} h^{2}+2 c_{3}$. It is shown that the genus of $\overline{\mathcal{C}}$ is 5 . Moreover, the curve $\mathcal{H}$ is an unramified double cover $\mathcal{H} \longrightarrow \mathcal{E},(w, h) \longmapsto(\zeta, \xi)=\left(w h, h^{2}\right)$, of an elliptic curve $\mathcal{E}$,

$$
\mathcal{E}: \zeta^{2}=\left(\left(\xi-c_{1}\right)^{2}+4\left(c_{3}-c_{4}\right)\right) \xi^{2}-4 c_{2} \xi
$$

Moreover, the curve $\mathcal{C}$ can be seen as an unramified double cover $\mathcal{C} \longrightarrow \mathcal{S},(z, h) \longmapsto(z, \xi)=\left(z, h^{2}\right)$, of a new curve $\mathcal{S}$, defined by

$$
\mathcal{S}: z^{4}+\left(h^{4}-c_{1} h^{2}+2 c_{3}\right) z^{2}+c_{4} h^{4}+\left(c_{2}^{2}-c-1 c-3\right) h^{2}+c_{3}^{2}=0 .
$$

The curve $\mathcal{S}$ itself is a double cover of the elliptic curve $\mathcal{E}$,

$$
\mathcal{S} \longrightarrow \mathcal{E}, \quad(z, \xi) \longmapsto(\zeta, \xi)=\left(2 z^{2}+\xi^{2}-c_{1} \xi+2 c_{3}, \xi\right)
$$

nd the genus of $\overline{\mathcal{S}}$ is equal to 3 . Finally, it is easily verified that the linearization of the flow is done on the Jacobian variety of the curve $\mathcal{C}$. 
The Goryachev-Chaplygin top : The differential equations of the Goryachev-Chaplygin top correspond to the case $I_{1}=I_{2}=4 I_{3}, l_{2}=l_{3}=0$ and are explicitly written in this case in the form (without restricting generality, values have been given to the constants in order not to weigh down the notations),

$$
\begin{aligned}
& \dot{m}_{1}=3 m_{2} m_{3}, \quad \dot{\gamma}_{1}=4 m_{3} \gamma_{2}-m_{2} \gamma_{3}, \\
& \dot{m}_{2}=-3 m_{1} m_{3}-4 \gamma_{3}, \quad \dot{\gamma}_{2}=m_{1} \gamma_{3}-4 m_{3} \gamma_{1} \\
& \dot{m}_{3}=4 \gamma_{2}, \quad \dot{\gamma}_{3}=m_{2} \gamma_{1}-m_{1} \gamma_{2} .
\end{aligned}
$$

This system admits the following four invariants :

$$
\begin{aligned}
& H_{1}=m_{1}^{2}+m_{2}^{2}+4 m_{3}^{2}-8 \gamma_{1}=6 b_{1}, \\
& H_{2}=\left(m_{1}^{2}+m_{2}^{2}\right) m_{3}+4 m_{1} \gamma_{3}=2 b_{2}, \\
& H_{3}=\gamma_{1}^{2}+\gamma_{2}^{2}+\gamma_{3}^{2}=b_{3}, \\
& H_{4}=m_{1} \gamma_{1}+m_{2} \gamma_{2}+m_{3} \gamma_{3}=0
\end{aligned}
$$

where $b_{1}, b_{2}, b_{3}$ are generic constants. The system (13) is integrable in the sense of Liouville, $H_{1}$ (the Hamiltonian) and $H_{4}$ are in involution while $H_{2}, H_{3}$ are Casimir invariants. The differential system (13) has a pair of Lax of the form

$$
\dot{A}=[A, B],
$$

with

$$
A=\left(\begin{array}{ccc}
0 & \gamma_{3} & \frac{1}{2} x_{1} h \\
-\gamma_{3} & -m_{3} h & \Gamma_{1}-h^{2} \\
\frac{1}{2} x_{2} h & -\Gamma_{2}+h^{2} & m_{3} h
\end{array}\right), \quad B=\left(\begin{array}{ccc}
3 i m_{3} & 0 & -i x_{1} \\
0 & 2 i m_{3} & 2 i h \\
-i x_{2} & -2 i h & -2 i m_{3}
\end{array}\right),
$$

where $x_{1}=m_{1}+i m_{2}, x_{2}=m_{1}-i m_{2}, \Gamma_{1}=\gamma_{1}+i \gamma_{2}, \Gamma_{1}=\gamma_{1}-i \gamma_{2}$. The transformation

$$
\left(t, m_{1}, m_{2}, m_{3}, \gamma_{1}, \gamma_{2}, \gamma_{3}\right) \longmapsto\left(-t,-m_{1},-m_{2},-m_{3},-\gamma_{1},-\gamma_{2},-\gamma_{3}\right),
$$

shows that the systems (13) and (14) are equivalent. The spectral curve, $\mathcal{C}: P(h, z)=\operatorname{det}(A-z I)=0$, is written explicitly

$$
\mathcal{C}: w^{3}+\left(h^{4}-\frac{3}{2} b_{1} h^{2}+b_{3}\right) w+\frac{b_{2}}{2} h^{3}=0,
$$

and one easily checks that the flow linearizes on the Jacobian of this curve.

\subsection{The Manakov geodesic flow on the group $S O(4)$}

Consider the group $S O(4)$ and its Lie algebra so(4) paired with itself, via the customary inner product

$$
\langle X, Y\rangle=-\frac{1}{2} \operatorname{tr}(X . Y)
$$

where

$$
X=\left(X_{i j}\right)_{1 \leq i, j \leq 4}=\sum_{i=1}^{6} x_{i} e_{i}=\left(\begin{array}{cccc}
0 & -x_{3} & x_{2} & -x_{4} \\
x_{3} & 0 & -x_{1} & -x_{5} \\
-x_{2} & x_{1} & 0 & -x_{6} \\
x_{4} & x_{5} & x_{6} & 0
\end{array}\right) \in s o(4) .
$$

A left invariant metric on $S O(4)$ is defined by a non-singular symmetric linear map $\Lambda: s o(4) \longrightarrow s o(4), X \longmapsto \Lambda . X$, and by the following inner product; given two vectors $g X$ and $g Y$ in the tangent space $S O(4)$ at the point $g \in S O(4)$,

$$
\langle g X, g Y\rangle=\left\langle X, \Lambda^{-1} . Y\right\rangle,
$$

regardless of $g$. Then the geodesic flow for this metric takes the following commutator form (Euler-Arnold equations) $:$

$$
\dot{X}=[X, \Lambda . X], \quad \equiv \frac{d}{d t}
$$


where

$$
\Lambda . X=\left(\lambda_{i j} X_{i j}\right)_{1 \leq i, j \leq 4}=\sum_{i=1}^{6} \lambda_{i} x_{i} e_{i}=\left(\begin{array}{cccc}
0 & -\lambda_{3} x_{3} & \lambda_{2} x_{2} & -\lambda_{4} x_{4} \\
\lambda_{3} x_{3} & 0 & -\lambda_{1} x_{1} & -\lambda_{5} x_{5} \\
-\lambda_{2} x_{2} & \lambda_{1} x_{1} & 0 & -\lambda_{6} x_{6} \\
\lambda_{4} x_{4} & \lambda_{5} x_{5} & \lambda_{6} x_{6} & 0
\end{array}\right) \in \operatorname{so}(4) .
$$

In view of the isomorphism between $\left(\mathbf{R}^{6}, \wedge\right)$, and $(s o(4),[]$,$) we write the system (15) as$

$$
\begin{aligned}
& \dot{x}_{1}=\left(\lambda_{3}-\lambda_{2}\right) x_{2} x_{3}+\left(\lambda_{6}-\lambda_{5}\right) x_{5} x_{6}, \\
& \dot{x}_{2}=\left(\lambda_{1}-\lambda_{3}\right) x_{1} x_{3}+\left(\lambda_{4}-\lambda_{4}\right) x_{4} x_{6}, \\
& \dot{x}_{3}=\left(\lambda_{2}-\lambda_{1}\right) x_{1} x_{2}+\left(\lambda_{5}-\lambda_{4}\right) x_{4} x_{5}, \\
& \dot{x}_{4}=\left(\lambda_{3}-\lambda_{5}\right) x_{3} x_{5}+\left(\lambda_{6}-\lambda_{2}\right) x_{2} x_{6}, \\
& \dot{x}_{5}=\left(\lambda_{4}-\lambda_{3}\right) x_{3} x_{4}+\left(\lambda_{1}-\lambda_{6}\right) x_{1} x_{6}, \\
& \dot{x}_{6}=\left(\lambda_{2}-\lambda_{4}\right) x_{2} x_{4}+\left(\lambda_{5}-\lambda_{1}\right) x_{1} x_{5} .
\end{aligned}
$$

This flow is Hamiltonian with regard to the usual Kostant-Kirillov symplectic structure induced on the orbit

$$
\mathcal{O}=\left\{A d_{g}^{*}(X)=g^{-1} X g: g \in S O(4)\right\},
$$

formed by the coadjoint action $A d_{g}^{*}(X)$ of the group $S O(4)$ on the dual Lie algebra $s o(4)^{*} \approx s o(4)$. Let $z_{1}, z_{2} \in \operatorname{so}(4)$ and consider $\xi_{1}=\left[X, z_{1}\right], \xi_{2}=\left[X, z_{2}\right]$ two tangent vectors to the orbit at the point $X \in \operatorname{so}(4)$. Then the symplectic structure is defined by

$$
\omega(X)\left(\xi_{1}, \xi_{2}\right)=\left\langle X,\left[z_{1}, z_{2}\right]\right\rangle .
$$

This orbit is 4-dimensional and is defined by setting two trivial quadratic invariants $H_{1}$ and $H_{2}$ equal to generic constants $c_{1}$ and $c_{2}$ :

$$
\begin{aligned}
& H_{1}=\sqrt{\operatorname{det} X}=x_{1} x_{4}+x_{2} x_{5}+x_{3} x_{6}=c_{1} \\
& H_{2}=-\frac{1}{2} \operatorname{tr}\left(X^{2}\right)=x_{1}^{2}+x_{2}^{2}+\cdots+x_{6}^{2}=c_{2}
\end{aligned}
$$

Fonctions $H$ defined on the orbit lead to Hamiltonian vector fields

$$
\dot{X}=[X, \nabla H] .
$$

In particular

$$
H=\frac{1}{2}\langle X, \Lambda X\rangle=\frac{1}{2}\left(\lambda_{1} x_{1}^{2}+\lambda_{2} x_{2}^{2}+\cdots+\lambda_{6} x_{6}^{2}\right),
$$

induces geodesic motion (15) The constants of the motion are given by the two quadratic invariants $H_{1}(17), H_{2}(18)$ and the Hamiltonian $H(17)$. Since the system is Hamiltonian on a 4-dimensional symplectic manifold

$$
\left\{H_{1}=c_{1}\right\} \cap\left\{H_{2}=c_{2}\right\},
$$

to make it completely integrable, one needs one independent invariant.

Note that the equations (16) can be written as a Hamiltonian vector field in another form

$$
\dot{x}(t)=J \frac{\partial H}{\partial x}, x \in \mathbf{R}^{6},
$$

with

$$
J=\left(\begin{array}{cccccc}
0 & -x_{3} & x_{2} & 0 & -x_{6} & x_{5} \\
x_{3} & 0 & -x_{1} & x_{6} & 0 & -x_{4} \\
-x_{2} & x_{1} & 0 & -x_{5} & x_{4} & 0 \\
0 & -x_{6} & x_{5} & 0 & -x_{3} & x_{2} \\
x_{6} & 0 & -x_{4} & x_{3} & 0 & -x_{1} \\
-x_{5} & x_{4} & 0 & -x_{2} & x_{1} & 0
\end{array}\right) \in s o(6)
$$


Since $\operatorname{det} J=0$, then $m=2 n+k$ and $m-k=r k J$. Here $m=6$ and $r g J=4$, then $n=k=2$. In summary, the system (16) has beside the energy $H$ (19), two trivial constants of motion $H_{1}(17)$ and $H_{2}$ (18) because :

$$
J \frac{\partial H_{2}}{\partial x}=J \frac{\partial H_{3}}{\partial x}=0 .
$$

and in order that the hamiltonian system (16) be completely integrable, it is suffices to have one more integral, which we take of the form

$$
H_{4}=\frac{1}{2}\left(\mu_{1} x_{1}^{2}+\mu_{2} x_{2}^{2}+\cdots+\mu_{6} x_{6}^{2}\right) .
$$

The four invariants must be functionally independent and in involution, so in particular

$$
\left\{H_{4}, H_{3}\right\}=\left\langle\frac{\partial H_{4}}{\partial x}, J \frac{\partial H_{3}}{\partial x}\right\rangle=0,
$$

i.e.,

$$
\begin{aligned}
& \left(\left(\lambda_{3}-\lambda_{2}\right) \mu_{1}+\left(\lambda_{1}-\lambda_{3}\right) \mu_{2}+\left(\lambda_{2}-\lambda_{1}\right) \mu_{3}\right) x_{1} x_{2} x_{3} \\
& +\left(\left(\lambda_{6}-\lambda_{5}\right) \mu_{1}+\left(\lambda_{1}-\lambda_{6}\right) \mu_{5}+\left(\lambda_{5}-\lambda_{1}\right) \mu_{6}\right) x_{1} x_{5} x_{6} \\
& +\left(\left(\lambda_{4}-\lambda_{6}\right) \mu_{2}+\left(\lambda_{6}-\lambda_{2}\right) \mu_{4}+\left(\lambda_{2}-\lambda_{4}\right) \mu_{6}\right) x_{2} x_{4} x_{6} \\
& +\left(\left(\lambda_{5}-\lambda_{4}\right) \mu_{3}+\left(\lambda_{3}-\lambda_{5}\right) \mu_{4}+\left(\lambda_{4}-\lambda_{3}\right) \mu_{5}\right) x_{3} x_{4} x_{5}=0 .
\end{aligned}
$$

Then

$$
\begin{aligned}
& \left(\lambda_{3}-\lambda_{2}\right) \mu_{1}+\left(\lambda_{1}-\lambda_{3}\right) \mu_{2}+\left(\lambda_{2}-\lambda_{1}\right) \mu_{3}=0, \\
& \left(\lambda_{6}-\lambda_{5}\right) \mu_{1}+\left(\lambda_{1}-\lambda_{6}\right) \mu_{5}+\left(\lambda_{5}-\lambda_{1}\right) \mu_{6}=0, \\
& \left(\lambda_{4}-\lambda_{6}\right) \mu_{2}+\left(\lambda_{6}-\lambda_{2}\right) \mu_{4}+\left(\lambda_{2}-\lambda_{4}\right) \mu_{6}=0, \\
& \left(\lambda_{5}-\lambda_{4}\right) \mu_{3}+\left(\lambda_{3}-\lambda_{5}\right) \mu_{4}+\left(\lambda_{4}-\lambda_{3}\right) \mu_{5}=0 .
\end{aligned}
$$

Put

$$
\mathcal{A}=\left(\begin{array}{cccccc}
\lambda_{3}-\lambda_{2} & \lambda_{1}-\lambda_{3} & \lambda_{2}-\lambda_{1} & 0 & 0 & 0 \\
\lambda_{6}-\lambda_{5} & 0 & 0 & 0 & \lambda_{1}-\lambda_{6} & \lambda_{5}-\lambda_{1} \\
0 & \lambda_{4}-\lambda_{6} & 0 & \lambda_{6}-\lambda_{2} & 0 & \lambda_{2}-\lambda_{4} \\
0 & 0 & \lambda_{5}-\lambda_{4} & \lambda_{3}-\lambda_{5} & \lambda_{4}-\lambda_{3} & 0
\end{array}\right)
$$

The number of solutions of this system is equal to the number of columns of the matrix $\mathcal{A}$ minus the rank of $\mathcal{A}$.

- If $r k \mathcal{A}=4$, we have two solutions : $\mu_{i}=1$ lead to the invariant $H_{2}$ and $\mu_{i}=\lambda_{i}$ lead to the invariant $H_{3}$. This is unacceptable.

- If $r k \mathcal{A}=3$, each four-order minor of $\mathcal{A}$ is singular. Now

$$
\begin{aligned}
& \left(\begin{array}{cccc}
\lambda_{3}-\lambda_{2} & \lambda_{1}-\lambda_{3} & \lambda_{2}-\lambda_{1} & 0 \\
\lambda_{6}-\lambda_{5} & 0 & 0 & 0 \\
0 & \lambda_{4}-\lambda_{6} & 0 & \lambda_{6}-\lambda_{2} \\
0 & 0 & \lambda_{5}-\lambda_{4} & \lambda_{3}-\lambda_{5}
\end{array}\right)=-\left(\lambda_{6}-\lambda_{5}\right) C, \\
& \left(\begin{array}{cccc}
\lambda_{1}-\lambda_{3} & \lambda_{2}-\lambda_{1} & 0 & 0 \\
0 & 0 & 0 & \lambda_{1}-\lambda_{6} \\
\lambda_{4}-\lambda_{6} & 0 & \lambda_{6}-\lambda_{2} & 0 \\
0 & \lambda_{5}-\lambda_{4} & \lambda_{3}-\lambda_{5} & \lambda_{4}-\lambda_{3}
\end{array}\right)=\left(\lambda_{1}-\lambda_{6}\right) C, \\
& \left(\begin{array}{cccc}
\lambda_{2}-\lambda_{1} & 0 & 0 & 0 \\
0 & 0 & \lambda_{1}-\lambda_{6} & \lambda_{5}-\lambda_{1} \\
0 & \lambda_{6}-\lambda_{2} & 0 & \lambda_{2}-\lambda_{4} \\
\lambda_{5}-\lambda_{4} & \lambda_{3}-\lambda_{5} & \lambda_{4}-\lambda_{3} & 0
\end{array}\right)=-\left(\lambda_{2}-\lambda_{1}\right) C,
\end{aligned}
$$

where

$$
\begin{aligned}
C \equiv \quad & \lambda_{1} \lambda_{6} \lambda_{4}+\lambda_{1} \lambda_{2} \lambda_{5}-\lambda_{1} \lambda_{2} \lambda_{4}+\lambda_{3} \lambda_{6} \lambda_{5}-\lambda_{3} \lambda_{6} \lambda_{4}-\lambda_{3} \lambda_{2} \lambda_{5} \\
& +\lambda_{4} \lambda_{2} \lambda_{5}+\lambda_{4} \lambda_{1} \lambda_{3}-\lambda_{4} \lambda_{1} \lambda_{5}+\lambda_{6} \lambda_{2} \lambda_{3}-\lambda_{6} \lambda_{2} \lambda_{5}-\lambda_{1} \lambda_{6} \lambda_{3},
\end{aligned}
$$


and it follows that the condition for which these minors are zero is $C=0$. Notice that this relation holds by cycling the indices : $1 \rightarrow 4,2 \rightarrow 5,3 \rightarrow 6$. Under Manakov [42] conditions,

$$
\begin{aligned}
& \lambda_{1}=\frac{\beta_{2}-\beta_{3}}{\alpha_{2}-\alpha_{3}}, \lambda_{2}=\frac{\beta_{1}-\beta_{3}}{\alpha_{1}-\alpha_{3}}, \lambda_{3}=\frac{\beta_{1}-\beta_{2}}{\alpha_{1}-\alpha_{2}}, \\
& \lambda_{4}=\frac{\beta_{1}-\beta_{4}}{\alpha_{1}-\alpha_{4}}, \lambda_{5}=\frac{\beta_{2}-\beta_{4}}{\alpha_{2}-\alpha_{4}}, \quad \lambda_{6}=\frac{\beta_{3}-\beta_{4}}{\alpha_{3}-\alpha_{4}},
\end{aligned}
$$

where $\alpha_{i}, \beta_{i} \in \mathbf{C}, \prod_{i<j}\left(\alpha_{i}-\beta_{j}\right) \neq 0$, equations (16) admits a Lax equation with an indeterminate $h$ :

$$
\begin{gathered}
(\overbrace{X+\alpha h})=[X+\alpha h, \Lambda X+\beta h], \\
\alpha=\left(\begin{array}{cccc}
\alpha_{1} & 0 & 0 & 0 \\
0 & \alpha_{2} & 0 & 0 \\
0 & 0 & \alpha_{3} & 0 \\
0 & 0 & 0 & \alpha_{4}
\end{array}\right), \quad \beta=\left(\begin{array}{cccc}
\beta_{1} & 0 & 0 & 0 \\
0 & \beta_{2} & 0 & 0 \\
0 & 0 & \beta_{3} & 0 \\
0 & 0 & 0 & \beta_{4}
\end{array}\right) . \\
\dot{\mathbb{X}} \\
\dot{X}=[X, \Lambda . X] \Leftrightarrow(15), \\
{[\alpha, \beta]=0 \text { trivially satisfied for diagonal matrices. }}
\end{gathered}
$$

The parameters $\mu_{1}, \ldots, \mu_{6}$ can be parameterized (like $\lambda_{1}, \ldots, \lambda_{6}$ ) by :

$$
\begin{aligned}
& \mu_{1}=\frac{\gamma_{2}-\gamma_{3}}{\alpha_{2}-\alpha_{3}}, \mu_{2}=\frac{\gamma_{1}-\gamma_{3}}{\alpha_{1}-\alpha_{3}}, \mu_{3}=\frac{\gamma_{1}-\gamma_{2}}{\alpha_{1}-\alpha_{2}} \\
& \mu_{4}=\frac{\gamma_{1}-\gamma_{4}}{\alpha_{1}-\alpha_{4}}, \mu_{5}=\frac{\gamma_{2}-\gamma_{4}}{\alpha_{2}-\alpha_{4}}, \mu_{6}=\frac{\gamma_{3}-\gamma_{4}}{\alpha_{3}-\alpha_{4}} .
\end{aligned}
$$

To use the method of isospectral deformations, consider the Kac-Moody extension $(n=4)$ :

$$
\mathcal{L}=\widetilde{g l(n, \mathbf{R})}=\left\{\sum_{-\infty}^{N} A_{i} h^{i}: N \text { arbitrary } \in \mathbf{Z}, A_{i} \in g l(n, \mathbf{R})\right\}
$$

of $g l(n, \mathbf{R})$ with the bracket :

$$
[A(h), B(h)]=\left[\sum A_{i} h^{i}, \sum B_{j} h^{j}\right]=\sum_{k}\left(\sum_{i+j=k}\left[A_{i}, B_{j}\right]\right) h^{k},
$$

and the nondegenerate, invariant inner product

$$
\langle A(h), B(h)\rangle=\left\langle\sum A_{i} h^{i}, \sum B_{j} h^{j}\right\rangle=\sum_{i+j=-1}\left\langle A_{i}, B_{j}\right\rangle,
$$

where $\langle$,$\rangle is the usual form defined on g l(n, \mathbf{R})$.

This Lie algebra has a natural decomposition

$$
\mathcal{L}=\mathcal{L}_{-\infty,-1}+\mathcal{L}_{0, \infty}, \quad \mathcal{L}_{i j}=\left\{\sum_{i \geq 0} A_{k} h^{k}\right\}
$$

Observe that $\mathcal{L}_{-\infty,-1}^{\perp}=\mathcal{L}_{-\infty,-1}$ and $\mathcal{L}_{0, \infty}^{\perp}=\mathcal{L}_{0, \infty}$ where $\perp$ is taken with respect the form above. The infinitedimensional Lie group underlying $\mathcal{L}_{-\infty,-1}$ acts coadjointly on the dual Kac-Moody Lie algebra $\mathcal{L}_{-\infty,-1}^{*} \approx \mathcal{L}_{0, \infty}^{\perp}=$ 
$\mathcal{L}_{0, \infty}$, according to the rule of customary conjugation followed by registering the non-negative powers of $h$ only. The orbits described in this way come equipped with a symplectic structure with Poisson bracket

$$
\left\{H_{1}, H_{2}\right\}(\alpha)=\left\langle\alpha,\left[\nabla_{\mathcal{L}_{-\infty,-1}^{*}} H_{1}, \nabla_{\mathcal{L}_{-\infty,-1}^{*}} H_{2}\right]\right\rangle
$$

where $\alpha \in \mathcal{L}_{-\infty,-1}^{*}$ and $\nabla_{\mathcal{L}_{-\infty,-1}^{*}} H \in \mathcal{L}_{-\infty,-1}$. The functions defined on this orbit are all in involution and the flow (21) evolves on the coadjoint orbit through the point $X+a h \in \mathcal{L}_{0, \infty}, X \in \operatorname{so}(4)$.

According to the Adler-Kostant-Symes theorem (where $\mathcal{K}=\mathcal{L}_{0, \infty}$ and $\mathcal{N}=\mathcal{L}_{-\infty,-1}$ are respectively the $\geq 0$ and $<0$ powers of $h$ in $\mathcal{L}$ ), the flow $(21)$ is hamiltonian on an orbit through the point $X+a h, X \in s o(4))$ formed by the coadjoint action of the subgroup $G_{\mathcal{N}} \subset S L(n)$ of lower triangular matrices on the dual Kac-Moody algebra $\mathcal{L}_{-\infty,-1}^{*} \approx \mathcal{L}_{0, \infty}^{\perp}=\mathcal{L}_{0, \infty}$. As a consequence, the coefficients of $z^{i} h^{i}$ appearing in curve :

$$
\mathcal{C}:\left\{(z, h) \in \mathbf{C}^{2}: \operatorname{det}(X+a h-z I)=0\right\},
$$

associated to the equation (21) are invariant of the system in involution for the symplectic structure of this orbit. Notice that

$$
\begin{gathered}
\operatorname{det}\left(g X g^{-1}\right)=\operatorname{det} X=\left(x_{1} x_{4}+x_{2} x_{5}+x_{3} x_{6}\right)^{2}, \\
\operatorname{tr}\left(g X g^{-1}\right)^{2}=\operatorname{tr}\left(g X^{2} g^{-1}\right)=\operatorname{tr}\left(X^{2}\right)=-2\left(x_{1}^{2}+x_{2}^{2}+\cdots+x_{6}^{2}\right) .
\end{gathered}
$$

Also the complex flows generated by these invariants can be realized as straight lines on the Abelian variety defined by the periods of the curve $\mathcal{C}$. Explicitly, equation (22) looks as follows

$$
\mathcal{C}: \quad \prod_{i=1}^{4}\left(\alpha_{i} h-z\right)+2 H_{4} h^{2}-2 H_{1} z h+2 H_{2} z^{2}+H_{3}^{2}=0,
$$

where

$$
H_{1}(X)=c_{1}, H_{2}(X)=c_{2}, H_{3}(X)=2 H=c_{3}, H_{4}(X)=c_{4},
$$

with $c_{1}, c_{2}, c_{3}, c_{4}$ generic constants. $\mathcal{C}$ is a curve of genus 3 and it has a natural involution $\sigma: \mathcal{C} \longrightarrow \mathcal{C},(z, h) \longmapsto$ $(-z,-h)$. Therefore the jacobian variety $\operatorname{Jac}(\mathcal{C})$ of $\mathcal{C}$ splits up into an even and old part : the even part is an elliptic curve $\mathcal{C}_{0}=\mathcal{C} / \sigma$ and the odd part is a 2 -dimensional Abelian surface $\operatorname{Prym}\left(\mathcal{C} / \mathcal{C}_{0}\right)$ (which is also noted, $\operatorname{Prym} \sigma(\mathcal{C})$ ) called the Prym variety :

$$
\operatorname{Jac}(\mathcal{C})=\mathcal{C}_{0}+\operatorname{Prym}_{\sigma}(\mathcal{C})
$$

The van Moerbeke-Mumford linearization method provides then an algebraic map from the complex affine variety $\bigcap_{i=1}^{4}\left\{H_{i}(X)=c_{i}\right\} \subset \mathbf{C}^{6}$ to the Jacobi variety $\operatorname{Jac}(\mathcal{C})$. By the antisymmetry of $\mathcal{C}$, this map sends this variety to the Prym variety $\operatorname{Prym}_{\sigma}(\mathcal{C})$ :

$$
\bigcap_{i=1}^{4}\left\{H_{i}(X)=c_{i}\right\} \rightarrow \operatorname{Prym}_{\sigma}(\mathcal{C}), \quad p \mapsto \sum_{k=1}^{3} s_{k},
$$

and the complex flows generated by the constants of the motion are straight lines on $\operatorname{Prym}_{\sigma}(\mathcal{C})$. Finally, we have the

Theorem 4.3. The geodesic flow (15) is a hamiltonian system with

$$
H \equiv H_{1}=\frac{1}{2}\left(\lambda_{1} x_{1}^{2}+\lambda_{2} x_{2}^{2}+\cdots+\lambda_{6} x_{6}^{2}\right),
$$

the hamiltonian. It has two trivial invariants

$$
\begin{aligned}
& H_{2}=\frac{1}{2}\left(x_{1}^{2}+x_{2}^{2}+\cdots+x_{6}^{2}\right) \\
& H_{3}=x_{1} x_{4}+x_{2} x_{5}+x_{3} x_{6} .
\end{aligned}
$$

Moreover, if

$$
\begin{aligned}
& \lambda_{1} \lambda_{6} \lambda_{4}+\lambda_{1} \lambda_{2} \lambda_{5}-\lambda_{1} \lambda_{2} \lambda_{4}+\lambda_{3} \lambda_{6} \lambda_{5}-\lambda_{3} \lambda_{6} \lambda_{4}-\lambda_{3} \lambda_{2} \lambda_{5} \\
& +\lambda_{4} \lambda_{2} \lambda_{5}+\lambda_{4} \lambda_{1} \lambda_{3}-\lambda_{4} \lambda_{1} \lambda_{5}+\lambda_{6} \lambda_{2} \lambda_{3}-\lambda_{6} \lambda_{2} \lambda_{5}-\lambda_{1} \lambda_{6} \lambda_{3}=0
\end{aligned}
$$


the system (16) has a fourth independent constant of the motion of the form

$$
H_{4}=\frac{1}{2}\left(\mu_{1} x_{1}^{2}+\mu_{2} x_{2}^{2}+\cdots+\mu_{6} x_{6}^{2}\right) .
$$

Then the system (16) is completely integrable and can be linearized on the Prym variety Prym $\operatorname{Pr}_{\alpha}(\mathcal{C})$.

The well-known Kirchhoff's equations of motion of a solid in an ideal fluid can be regarded as the equations of the geodesics of the right-invariant metric on the group $E(3)=S O(3) \times \mathbf{R}^{3}$ of motions of 3-dimensional euclidean space $\mathbf{R}^{3}$, generated by rotations and translations. Hence the motion has the trivial coadjoint orbit invariants $\langle p, p\rangle$ and $\langle p, l\rangle$. As it turns out, this is a special case of a more general system of equations written as

$$
\dot{x}=x \wedge \frac{\partial H}{\partial x}+y \wedge \frac{\partial H}{\partial y}, \quad \dot{y}=y \wedge \frac{\partial H}{\partial x}+x \wedge \frac{\partial H}{\partial y},
$$

where $x=\left(x_{1}, x_{2}, x_{3}\right) \in \mathbf{R}^{3}$ and $y=\left(y_{1}, y_{2}, y_{3}\right) \in \mathbf{R}^{3}$. The first set can be obtained from the second by putting $(x, y)=(l, p / \varepsilon)$ and letting $\varepsilon \rightarrow 0$. The latter set of equations is the geodesic flow on $S O(4)$ for a left invariant metric defined by the quadratic form $H$.

\subsection{Jacobi's geodesic flow on an ellipsoid and Neumann's problem}

For $m=2$, i.e., $V_{2}$, if one chooses

$$
A=\alpha h^{2}-h x \wedge y-y \otimes y \quad x, y \in \mathbf{R}^{n},
$$

(which can also be considered as a rank 2 perturbation of the diagonal matrix $\alpha$, see $[2,3,44,45])$ then equation $(7)$ reduces to

$$
\dot{A}=\left[A, a d_{\beta} a d_{\alpha}^{-1}(y \wedge x)+\beta h\right], \quad \beta_{i}=f^{\prime}\left(\alpha_{i}\right) .
$$

This equation can be reduced to the following Hamiltonian system :

$$
\begin{aligned}
& \dot{x}=-\left(a d_{\beta} a d_{\alpha}^{-1}(y \wedge x)\right) x-\beta y=-\frac{\partial H_{\beta}}{\partial y}, \\
& \dot{y}=-\left(a d_{\beta} a d_{\alpha}^{-1}(y \wedge x)\right) y=\frac{\partial H_{\beta}}{\partial x},
\end{aligned}
$$

where

$$
H_{\beta}=\frac{1}{2} \sum_{i} \beta_{i}\left(y_{i}^{2}+\sum_{j \neq i} \frac{\left(x_{i} y_{j}-x_{j} y_{i}\right)^{2}}{\alpha_{i}-\alpha_{j}}\right)
$$

which

- for $f(z)=\ln z$, i.e., $\beta_{i}=\frac{1}{\alpha_{i}}$, we obtain the problem of Jacobi's geodesic flow on the ellipsoid

$$
\frac{x_{1}^{2}}{\alpha_{1}^{2}}+\cdots+\frac{x_{n}^{2}}{\alpha_{n}^{2}}=1
$$

expressing the motion of the tangent line $x+s y: s \in \mathbf{R}$ to the ellipsoid in the direction $y$ of the geodesic.

- for $f(z)=\frac{1}{2} z^{2}$, i.e., $\beta_{i}=\alpha_{i}$, we get the Neumann's motion [47] of a point on the sphere $S^{n-1},|x|=1$, under the influence of the force $-\alpha x$.

From Theorem 3.3, both motions are straight lines on $J a c(\mathcal{C})$, where $\mathcal{C}$ turns out to be hyperelliptic of genus $n-1$ (much lower than the generic one) ramified at the following $2 n$ points : some point at $\infty$, the $n$ points $\alpha_{i}$ and $n-1$ other points $\lambda_{i}$ of geometrical significance, based on the observation that generically a line in $\mathbf{R}^{n}$ touches $n-1$ confocal quadrics. To be precise, the set of all common tangent lines to $n-1$ confocal quadrics

$$
Q_{\lambda_{i}}(x, x)+l=0, \quad i=1, \ldots, n-1,
$$

where $Q_{u}(x, y)=\left\langle(u-\alpha)^{-1} x, y\right\rangle$, can be parametrized by the quotient of the Jacobian of the hyperelliptic curve $\mathcal{C}$ above by an abelian group $G$. The group is generated by the discrete action obtained by flipping the signs of $x_{k}$ and 
$y_{k}$ and some trivial one-dimensional action. Letting $h \rightarrow 0$ in the matrx $A$ and excising the largest eigenvalue from this matrix leads to a new isospectral symmetric matrix

$$
L=\left(I-P_{y}\right)(\alpha-x \otimes x)\left(I-P_{y}\right),
$$

and a flow

$$
\dot{L}=\left[\operatorname{ad}_{\beta} a d_{\alpha}^{-1} x \wedge y, L\right]
$$

where the spectrum of $L$ is given by the $n-1$ branch points $\lambda_{i}$ above and zero. From these considerations, it follows that the tangent line $\{x+s y: s \in \mathbf{R}\}$ to the ellipsoid remains tangent to $n-2$ other confocal quadrics and the corresponding $n-1$ eigenfunctions of $L$ provide the orthogonal set of normals to the $n-1$ quadrics at the points of tangency, hence recovering a theorem of Chasles. The close relationship between Jacobi's and Neumann's problems, which in fact live on the same orbits, was implemented by Knörrer [26, 27], who showed that the normal vector to the ellipsoid moves according to the Neumann problem, when the point moves according to the geodesic. Also the set of all $n-1$ dimensional linear subspaces in the intersection of two quadrics

$$
\begin{array}{r}
X_{1}^{2}+\cdots+X_{n}^{2}-Y_{1}^{2}-\cdots-Y_{n-1}^{2}=0, \\
\alpha_{1} X_{1}^{2}+\cdots+\alpha_{n} X_{n}^{2}-\lambda_{1} Y_{1}^{2}-\cdots-\lambda_{n-1} Y_{n-1}^{2}=X_{0}^{2},
\end{array}
$$

in $\mathbf{P}^{2 n-1}$ is the Jacobian of the curve $\mathcal{C}$ defined above. This is done by observing that the set of linear subspaces in the above quadrics is the same as the set of $(n-2)$-dimensional linear subspaces tangent to $n-1$ quadrics

$$
\left(\alpha_{1}-\lambda_{j}\right) X_{1}^{2}+\cdots+\left(\alpha_{n}-\lambda_{j}\right) X_{n}^{2}=X_{0}^{2}, \quad j=1,2, \ldots, n-1
$$

which is dual to the set of tangents to the confocal quadrics. The Neumann problem is also strikingly related to the Korteweg-de Vries (KdV) equation and various other nonlinear partial differential equations.

\subsection{A family of integrable systems}

We consider the hamiltonian

$$
H=\frac{1}{2}\left(x_{1}^{2}+x_{2}^{2}+a_{1} y_{1}^{2}+a_{2} y_{2}^{2}\right)+\frac{1}{4} y_{1}^{4}+\frac{1}{4} a_{3} y_{2}^{4}+\frac{1}{2} a_{4} y_{1}^{2} y_{2}^{2},
$$

where $a_{1}, a_{2}, a_{3}, a_{4}$ are arbitrary constants. The corresponding system is given by

$$
\begin{aligned}
\ddot{y}_{1}+\left(a_{1}+y_{1}^{2}+a_{4} y_{2}^{2}\right) y_{1} & =0, \\
\ddot{y}_{2}+\left(a_{2}+a_{3} y_{2}^{2}+a_{4} y_{1}^{2}\right) y_{2} & =0 .
\end{aligned}
$$

The integrability of this hamiltonian system has been studied by several authors. It arises in connection with some problems in scalar field theory and in the semi-classical method in quantum field theory. It corresponds to the Garnier system and to the anisotropic harmonic oscillator in a radial quartic potential. Also, this hamiltonian can be connected to the coupled nonlinear Schrödinger equations. This section deals with the problem of integrability of the system (25) corresponding to the choice : $a_{1}, a_{2}$ arbitrary constants and $a_{3}=a_{4}=1$, i.e.,

$$
H=\frac{1}{2}\left(x_{1}^{2}+x_{2}^{2}+a_{1} y_{1}^{2}+a_{2} y_{2}^{2}\right)+\frac{1}{4}\left(y_{1}^{2}+y_{2}^{2}\right)^{2} .
$$

The corresponding system is given by

$$
\begin{aligned}
& \ddot{y}_{1}+\left(a_{1}+y_{1}^{2}+y_{2}^{2}\right) y_{1}=0 \\
& \ddot{y}_{2}+\left(a_{2}+y_{2}^{2}+y_{1}^{2}\right) y_{2}=0 .
\end{aligned}
$$

For $a_{1}=a_{2}$, it is easy to show that the problem can be integrated in terms of elliptic functions. We suppose that $a_{1} \neq a_{2}$.

Using the results given in [12], we consider the Lax representation in the form

$$
\dot{A}_{h}=\left[B_{h}, A_{h}\right]=B_{h} A_{h}-A_{h} B_{h}, \quad \cdot \equiv \frac{\partial}{\partial t}
$$


with the following ansatz for the Lax operator

$$
A_{h}=\left(\begin{array}{cc}
u(h) & v(h) \\
w(h) & -u(h)
\end{array}\right), \quad B_{h}=\left(\begin{array}{cc}
0 & 1 \\
r(h) & 0
\end{array}\right)
$$

where

$$
\begin{aligned}
v(h) & =-\left(a_{1}+h\right)\left(a_{2}+h\right)\left(1+\frac{1}{2}\left(\frac{y_{1}^{2}}{a_{1}+h}+\frac{y_{2}^{2}}{a_{2}+h}\right)\right), \\
u(h) & =\frac{1}{2}\left(a_{1}+h\right)\left(a_{2}+h\right)\left(\frac{x_{1} y_{1}}{a_{1}+h}+\frac{x_{2} y_{2}}{a_{2}+h}\right), \\
w(h) & =\left(a_{1}+h\right)\left(a_{2}+h\right)\left(\frac{1}{2}\left(\frac{x_{1}^{2}}{a_{1}+h}+\frac{x_{2}^{2}}{a_{2}+h}\right)-h+\frac{1}{2}\left(y_{1}^{2}+y_{2}^{2}\right)\right), \\
r(h) & =h-y_{1}^{2}-y_{2}^{2} .
\end{aligned}
$$

The system (25) admits a Lax representation given by (28). The proof is straightforward and based on direct computation : we have

$$
\left[B_{h}, A_{h}\right]=\left(\begin{array}{cc}
w(h)-v(h) r(h) & -2 u(h) \\
2 u(h) r(h) & v(h) r(h)-w(h)
\end{array}\right),
$$

and it follows from (26), (27) and (29) that

$$
\begin{aligned}
\dot{u}(h) & =w(h)-v(h) r(h), \\
\dot{v}(h) & =-2 u(h), \\
\dot{w}(h) & =2 u(h) r(h) .
\end{aligned}
$$

Equation (28) means that for $h \in \mathbf{C}$ and under the time evolution of the system, $A_{h}(t)$ remain similar to $A_{h}(0)$. So the spectrum of $A_{h}$ is conserved, i.e., it undergoes an isospectral deformation. The eigenvalues of $A_{h}$, viewed as functionals, represent the integrals (constants of the motion) of the system. To be precise, a hamiltonian flow of the type (28) preserves the spectrum of $A_{h}$ and therefore its characteristic polynomial $\operatorname{det}\left(A_{h}-z I\right)$. We form the Riemann surface in $(z, h)$ space :

$$
\Gamma: \operatorname{det}\left(A_{h}-z I\right)=0,
$$

whose coefficients are functions of the phase space. Explicitly, this equation looks as follows

$$
\begin{aligned}
\Gamma: z^{2} & =u^{2}(h)+v(h) w(h) \equiv P_{5}(h), \\
& =\left(a_{1}+h\right)\left(a_{2}+h\right)\left(h^{3}+\left(a_{1}+a_{2}\right) h^{2}+\left(a_{1} a_{2}-H_{1}\right) h-H_{2}\right),
\end{aligned}
$$

where $H_{1}=H$ is defined by (26) with $a_{1}, a_{2}$ arbitrary, $a_{3}=a_{4}=1$ and a second quartic integral $H_{2}$ of the form :

$$
\begin{aligned}
H_{2}= & \frac{1}{4}\left(a_{2} y_{1}^{4}+a_{1} y_{2}^{4}+\left(a_{1}+a_{2}\right) y_{1}^{2} y_{2}^{2}+\left(x_{1} y_{2}-x_{2} y_{1}\right)^{2}\right) \\
& +\frac{1}{2}\left(a_{2} x_{1}^{2}+a_{1} x_{2}^{2}+a_{1} a_{2}\left(y_{1}^{2}+y_{2}^{2}\right)\right) .
\end{aligned}
$$

The curve $\Gamma$ determined by the fifth-order equation (30) is smooth, hyperelliptic and its genus is two. Obviously, $\Gamma$ is invariant under the involution $(h, z) \longmapsto(h,-z)$. The second hamiltonian vector field is written as

$$
\begin{aligned}
& \dot{y}_{1}=\frac{1}{2}\left(x_{1} y_{2}-x_{2} y_{1}\right) y_{2}+a_{2} x_{1}, \\
& \dot{y}_{2}=-\frac{1}{2}\left(x_{1} y_{2}-x_{2} y_{1}\right) y_{1}+a_{1} x_{2}, \\
& \dot{x}_{1}=-a_{2} y_{1}^{3}-\frac{1}{2}\left(a_{1}+a_{2}\right) y_{1} y_{2}^{2}+\frac{1}{2}\left(x_{1} y_{2}-x_{2} y_{1}\right) x_{2}-a_{1} a_{2} y_{1}, \\
& \dot{x}_{2}=-a_{1} y_{2}^{3}-\frac{1}{2}\left(a_{1}+a_{2}\right) y_{1}^{2} y_{2}-\frac{1}{2}\left(x_{1} y_{2}-x_{2} y_{1}\right) x_{1}-a_{1} a_{2} y_{2} .
\end{aligned}
$$

These vector fields are in involution with respect to the associated Poisson bracket. For generic $c=\left(c_{1}, c_{2}\right) \in C^{2}$,

$$
V_{c}=\left\{H_{1}=c_{1}, H_{2}=c_{2}\right\},
$$


is a smooth affine surface. The linearized flow could be realized on the jacobian variety $J a c(\Gamma)$ of the curve $(30)$. Indeed, as the structure of the matrices $A_{h}$ and $B_{h}$ (degree of the polynomials in $h$ ) is the same as for the well-known Neumann system, so both systems are linearized in the same way. We can construct an algebraic map from $V_{c}$ to the Jacobi variety $\operatorname{Jac}(\Gamma)$ :

$$
V_{c} \longrightarrow \operatorname{Jac}(\Gamma), \quad p \in V_{c} \longmapsto\left(s_{1}+s_{2}\right) \in \operatorname{Jac}(\Gamma),
$$

and the flows generated by the constants of the motion are straight lines on $J a c(\Gamma)$, i.e., the linearizing equations are given by

$$
\sum_{i=1}^{2} \int_{s_{i}(0)}^{s_{i}(t)} \omega_{k}=c_{k} t, \quad 0 \leq k \leq 2,
$$

where $\omega_{1}, \omega_{2}$ span the two-dimensional space of holomorphic differentials on the curve $\Gamma$ and $s_{1}, s_{2}$ two appropriate variables, algebraically related to the originally given ones, for which the Hamilton-Jacobi equation could be solved by separation of variables. Consequently, we have

Theorem 4.4. The system (23) is completely integrable for all $a_{1}, a_{2}, a_{3}=a_{4}=1$ (i.e., system (27)) and admits a Lax representation given by (28). The invariants of $A_{h}$ are integrals of motion in involution. The first integral is given by the hamiltonian $H_{1}=H$ (26) whereas the second integral $H_{2}$ is also quartic and has the form (31). The flows generated by $H_{1}$ and $H_{2}$ are straight line motions on the jacobian variety Jac $(\Gamma)$ of a smooth genus two hyperelliptic curve $\Gamma$ (30) associated to Lax equation (28).

We introduce coordinates $s_{1}$ and $s_{2}$ on the surface $V_{c}(32)$, such that $v\left(s_{1}\right)=v\left(s_{2}\right)=0, a_{1} \neq a_{2}$,

$$
y_{1}^{2}=2 \frac{\left(a_{1}+s_{1}\right)\left(a_{1}+s_{2}\right)}{a_{1}-a_{2}}, \quad y_{2}^{2}=2 \frac{\left(a_{2}+s_{1}\right)\left(a_{2}+s_{2}\right)}{a_{2}-a_{1}},
$$

i.e.,

$$
s_{1}+s_{2}=\frac{1}{2}\left(y_{1}^{2}+y_{2}^{2}\right)-a_{1}-a_{2}, \quad s_{1} s_{2}=-\frac{1}{2}\left(a_{2} y_{1}^{2}+a_{1} y_{2}^{2}\right)+a_{1} a_{2} .
$$

After some manipulations, we obtain the following equations :

$$
\dot{s}_{1}=2 \frac{\sqrt{P_{5}\left(s_{1}\right)}}{s_{1}-s_{2}}, \quad \dot{s}_{2}=2 \frac{\sqrt{P_{5}\left(s_{2}\right)}}{s_{2}-s_{1}},
$$

where $P_{5}(s)$ is defined by (30). These equations can be integrated by the Abelian mapping

$$
\Gamma \longrightarrow \operatorname{Jac}(\Gamma)=\mathbf{C}^{2} / L, \quad p \longmapsto\left(\int_{p_{0}}^{p} \omega_{1}, \int_{p_{0}}^{p} \omega_{2}\right)
$$

where the genus two hyperelliptic curve $\Gamma$ is given by the equation $(30), L$ is the lattice generated by the vectors $n_{1}+\Omega n_{2},\left(n_{1}, n_{2}\right) \in \mathbf{Z}^{2}, \Omega$ is the matrix of period of $\Gamma,\left(\omega_{1}, \omega_{2}\right)$ is a basis of holomorphic differentials on the curve Г, i.e.,

$$
\omega_{1}=\frac{d s}{\sqrt{P_{5}(s)}}, \quad \omega_{2}=\frac{s d s}{\sqrt{P_{5}(s)}},
$$

and $p_{0}$ is a fixed point. Consequently, we have

Theorem 4.5. The system of differential equations (27), can be integrated in terms of genus two hyperelliptic functions of time.

\subsection{The coupled nonlinear Schrödinger equations}

The system of two coupled nonlinear Schrödinger equations is given by

$$
\begin{aligned}
& i \frac{\partial a}{\partial z}+\frac{\partial^{2} a}{\partial t^{2}}+\Omega_{0} a+\frac{2}{3}\left(|a|^{2}+|b|^{2}\right) a+\frac{1}{3}\left(a^{2}+b^{2}\right) \bar{a}=0 \\
& i \frac{\partial b}{\partial z}+\frac{\partial^{2} b}{\partial t^{2}}-\Omega_{0} b+\frac{2}{3}\left(|a|^{2}+|b|^{2}\right) b+\frac{1}{3}\left(a^{2}+b^{2}\right) \bar{b}=0
\end{aligned}
$$


where $a(z, t)$ and $b(z, t)$ are functions of $z$ and $t$, the bar "-" denotes the complex conjugation, "|l" denotes the modulus and $\Omega_{0}$ is a constant. These equations play a significant role in mathematics, with a important number of physical applications. We seek solutions of (33) in the following form

$$
a(z, t)=y_{1}(t) \exp (i \Omega z), \quad b(z, t)=y_{2}(t) \exp (i \Omega z),
$$

where $y_{1}(t)$ et $y_{2}(t)$ are two functions and $\Omega$ is an arbitrary constant. Then we obtain the system

$$
\begin{aligned}
& \ddot{y}_{1}+\left(y_{1}^{2}+y_{2}^{2}\right) y_{1}=\left(\Omega-\Omega_{0}\right) y_{1}, \\
& \ddot{y}_{2}+\left(y_{1}^{2}+y_{2}^{2}\right) y_{2}=\left(\Omega+\Omega_{0}\right) y_{2} .
\end{aligned}
$$

The latter coincides obviously with $(27)$ for $a_{1}=\Omega_{0}-\Omega$ and $a_{2}=-\Omega_{0}-\Omega$.

\subsection{The Yang-Mills equations}

We consider the Yang-Mills system for a field with gauge group $S U(2)$ :

$$
D_{j} F_{j k}=\frac{\partial F_{j k}}{\partial \tau_{j}}+\left[A_{j}, F_{j k}\right]=0,
$$

where $F_{j k}, A_{j} \in T_{e} S U(2), 1 \leq j, k \leq 4$ and

$$
F_{j k}=\frac{\partial A_{k}}{\partial \tau_{j}}-\frac{\partial A_{j}}{\partial \tau_{k}}+\left[A_{j}, A_{k}\right] .
$$

The self-dual Yang-Mills (SDYM) equations is an universal system for which some reductions include all classical tops from Euler to Kowalewski (0+1-dimensions), K-dV, Nonlinear Schrdinger, Sine-Gordon, Toda lattice and N-waves equations (1+1-dimensions), KP and D-S equations (2+1-dimensions). In the case of homogeneous double-component field, we have $\partial_{j} A_{k}=0, j \neq 1, A_{1}=A_{2}=0, A_{3}=n_{1} U_{1} \in s u(2), A_{4}=n_{2} U_{2} \in s u(2)$ where $n_{i}$ are $s u(2)$-generators (i.e., they satisfy commutation relations : $\left.n_{1}=\left[n_{2},\left[n_{1}, n_{2}\right]\right], n_{2}=\left[n_{1},\left[n_{2}, n_{1}\right]\right]\right)$. The system becomes

$$
\frac{\partial^{2} U_{1}}{\partial t^{2}}+U_{1} U_{2}^{2}=0, \quad \frac{\partial^{2} U_{2}}{\partial t^{2}}+U_{2} U_{1}^{2}=0,
$$

with $t=\tau_{1}$. By setting $U_{j}=y_{j}, \frac{\partial U_{j}}{\partial t}=x_{j}, j=1,2$, Yang-Mills equations are reduced to Hamiltonian system

$$
\dot{x}=J \frac{\partial H}{\partial x}, \quad x=\left(y_{1}, y_{2}, x_{1}, x_{2}\right)^{\top}, \quad J=\left(\begin{array}{cc}
O & -I \\
I & O
\end{array}\right),
$$

with $H=\frac{1}{2}\left(x_{1}^{2}+x_{2}^{2}+y_{1}^{2} y_{2}^{2}\right)$, the Hamiltonian. The symplectic transformation

$$
\begin{aligned}
& x_{1} \longleftarrow \frac{\sqrt{2}}{2}\left(x_{1}+x_{2}\right), \quad x_{2} \longleftarrow \frac{\sqrt{2}}{2}\left(x_{1}-x_{2}\right), \\
& y_{1} \longleftarrow \frac{1}{2}(\sqrt[4]{2})\left(y_{1}+i y_{2}\right), \quad y_{2} \longleftarrow \frac{1}{2}(\sqrt[4]{2})\left(y_{1}-i y_{2}\right),
\end{aligned}
$$

takes this Hamiltonian into

$$
H=\frac{1}{2}\left(x_{1}^{2}+x_{2}^{2}\right)+\frac{1}{4} y_{1}^{4}+\frac{1}{4} y_{2}^{4}+\frac{1}{2} y_{1}^{2} y_{2}^{2},
$$

which coincides with (26) for $a_{1}=a_{2}=0$.

\section{References}

[1] M. Adler, "On a trace functional for pseudo-differential operators and the symplectic structure of the Kortewegde Vries equation", Invent. Math., Vol.50, (1979), pp.219-248. 
[2] M. Adler and P. van Moerbeke, "Completely integrable systems, Euclidean Lie algebras and curves", Adv. in Math., Vol.38, (1980), pp.267-317.

[3] M. Adler and P. van Moerbeke, "Linearization of Hamiltonian systems, Jacobi varieties and representation theory", Adv. in Math., Vol.38, (1980), pp.318-379.

[4] M. Adler and P. van Moerbeke, "The Kowalewski and Hénon-Heiles motions as Manakov geodesic flows on SO(4)- a two-dimensional family of Lax pairs", Commun. Math. Phys., Vol.113, (1988), pp.659-700.

[5] M. Adler and P. van Moerbeke, "The complex geometry of the Kowalewski-Painlevé analysis", Invent. Math., Vol.7, (1989), pp.3-51.

[6] M. Adler, P. van Moerbeke and P. Vanhaecke, Algebraic integrability, Painlevé geometry and Lie algebras. A series of modern surveys in mathematics, Volume 47, Springer-Verlag, (2004).

[7] G. Appel'rot, "The problem of motion of a rigid body about a fixed point", Uchenye Zap. Mosk. Univ. Otdel. Fiz. Mat. Nauk, Vol.11, No.3, (1894), pp.1-112.

[8] V.I. Arnold, Mathematical methods in classical mechanics, Springer, (1978).

[9] A.I. Belokolos, V.Z. Bobenko, V.Z. Enol'skii, A.R. Its and V.B. Matveev, Algebro-Geometric approach to nonlinear integrable equations, Springer-Verlag, (1994).

[10] D. Bobylev, "On a certain particular solution of the differential equations of rotation of a heavy rigid body about a fixed point", Trudy Otdel. Fiz. Nauk Obsc. Estestvozn., Vol.8, (1896), pp.2125.

[11] S.A. Chaplygin, "A new case of rotation of a rigid body, supported at one point", Gostekhizdat, Vol.I, (1948), pp.118-124.

[12] P.L. Christiansen, J.C. Eilbeck, V.Z. Enolskii and N.A. Kostov, "Quasi-periodic solutions of the coupled nonlinear Schrödinger equations", Proc. R. Soc. Lond., Vol.451, (1995), pp.685-700.

[13] B.A. Dubrovin and S.P. Novikov, "Periodic and conditionally periodic analogs of the many-soliton solutions of the Korteweg-de Vries equation", Dokl. Akad. Nauk URSS, Vol.6, (1974), pp.2131-2144.

[14] L. Euler, Theoria motus corporum solidorum seu rigidorum ex primis nostrae cognitionis principiis stabilita et ad omnes motus, qui in huiusmodi corpora cadere possunt, accomodata., Rostock und Greifswald Röse, (1765).

[15] C.S. Gardner, J.M. Greene, M.D. Kruskal and R.M. Miura, "Method for solving the Korteweg-de Vries equation", Journal name, Vol.19, (1967), pp.1095-1097.

[16] D. Goryachev, "On the motion of a rigid material body about a fixed point in the case $\mathrm{A}=\mathrm{B}=4 \mathrm{C}$ ", Journal name, Vol.21, No.3, (1900), pp.XX-XX.

[17] P.A. Griffiths and J. Harris, Principles of algebraic geometry, Wiley-Interscience, (1978).

[18] P.A. Griffiths, "Linearizing flows and a cohomological interpretation of Lax equations", Amer. J. of Math., Vol.107, (1985), pp.1445-1483.

[19] L. Haine, "Geodesic flow on SO(4) and Abelian surfaces", Math. Ann., Vol.263, (1983), pp.435-472.

[20] L. Haine and E. Horozov, "A Lax pair for Kowalevski’s top", Phys. D, Vol.29, (1987), pp.173-180.

[21] W. Hess, "Über die Euler'schen Bewegungsgleichungen und über eine neue particuläre Lösung des Problems der Bewegung eines starren Körpers un einen festen Punkt", Math. Ann., Vol.37, No.2, (1890), pp.178180.

[22] P.J. Holmes and J.E. Marsden, "Horseshoes and Arnold diffusion for Hamiltonian systems on Lie Groups", Indiana Univ. Math. J., Vol.32, (1983), pp.273-310.

[23] E. Husson, "Recherche des intégrales algébriques dans le mouvement d'un solide pesant autour d'un point fixe", Ann. Fac. Sc. Univ. Toulouse, Vol.8, No.2, (1906), pp.73-152.

[24] C. G. J. Jacobi, Vorlesungen über Dynamik, G. Reimer, (1884).

[25] G. Kirchoff, Vorlesungen ber Mathematische Physik, Vol. 1, Mechanik, Teubner, Leipzig, (1876).

[26] H. Knörrer, "Geodesics on the ellipsoïd", Invent. Math., Vol.59, (1980), pp.119-143. 
[27] H. Knörrer, "Geodesics on quadrics and a mechanical problem of C. Neumann", Reine und Angew. Math. J., Vol.334, (1980), pp.69-78.

[28] D.J. Korteweg and G. de Vries, "On the change of form of long waves advancing in a rectangular canal and on a new type of long stationary waves", Journal name, Vol.39, (1895), pp.422-443.

[29] B. Kostant, "The solution to a generalized Toda lattice and representation theory", Adv. in Math., Vol.34, (1979), pp.195-338.

[30] F. Kötter, "Uber die Bewegung eines festen Körpers in einer Flüssigkeit I, II", Journal fr die reine und angewandte Mathematik, Vol.109, (1892), pp.51-81, 89-111.

[31] S. Kowalewski, "Sur le problème de la rotation d'un corps solide autour d'un point fixe", Acta Math., Vol.12, (1889), pp.177-232.

[32] I.M. Krichever, "Algebraic-geometric construction of Zakhorov-Shabat equations and their periodic solutions", Sov. Math., Dokl., Vol.17, (1976), pp.394-397.

[33] J.L. Lagrange, Mécanique analytique, Oeuvres de Lagrange, Gauthier-Villars, (1888).

[34] P. Lax, "Integrals of nonlinear equations of evolution and solitary waves", Comm. Pure and Appl. Math., Vol.21, (1968), pp.467-490.

[35] A. Lesfari, "Abelian surfaces and Kowalewski's top", Ann. Scient. École Norm. Sup., Vol.4, No.21, (1988), pp.35-55.

[36] A. Lesfari, "Geodesic flow on SO(4), Kac-Moody Lie algebra and singularities in the complex t-plane", Publ. Mat., Barc., Vol.43, No.1, (1999), pp.261-279.

[37] A. Lesfari, "Integrables Hamiltonian systems and the isospectral deformation method", Int. J. of Appl. Math. and Mech., Vol.3, No.4, (2007), pp.35-55.

[38] A. Lesfari, "Integrable systems and complex geometry", Lobachevskii Journal of Mathematics, Vol.30, No.4, (2009), pp.292-326.

[39] A. Lesfari, "Algebraic integrability : the Adler-van Moerbeke approach", Regul. Chaotic Dyn., Vol.16, No.3-4, (2011), pp.187-209.

[40] A. Lesfari, Introduction à la géométrie algébrique complexe, Hermann, (2015).

[41] R. Liouville, "Sur le monvement d'un corps solide pesant suspendu par l'un de ses points", Acta Math., Vol.XX, (1896), pp.239-284.

[42] S.V. Manakov, "Remarks on the Integrals of the Euler Equations of the $n$-Dimensional Heavy Top", Fund. Anal. Appi.Fund. Anal. Appi., Vol.10, No.4, (1976), pp.93-94.

[43] H.P. Mc Kean and P. van Moerbeke, "The spectrum of Hill's equation", Invent. Math., Vol.30, (1975), pp.217274.

[44] J. Moser, "Three integrable Hamiltonian systems connected with isospectral deformations", Adv. Math., Vol.16, (1975), pp.197-219.

[45] J. Moser, "Geometry of quadrics and spectral theory", Chern Sympos., (1980), pp.147-188.

[46] D. Mumford, Tata lectures on theta I, II, Progress in Math., Birkhaüser, (1983).

[47] C. Neumann, "De problemate quodam mechanics, quod ad primam integralium ultraellipticorum classem revocatur", Reine und Angew. Math. J., Vol.56, (1859), pp.46-63.

[48] P. Painlevé, Oeuvres, tomes 1,2,3, Edition du C.N.R.S., (1975).

[49] A.M. Perelomov, Integrable systems of classical mechanics and Lie algebras, Birkhuser, (1990).

[50] H. Poincaré, Leons de mécanique céleste, 3 tomes, Gauthier-Villars, (1905-1910).

[51] L. Poinsot, "Théorie nouvelle de la rotation des corps", Journal de Liouville, Vol.16, (1851). 
[52] V.A. Steklov, "Über die Bewegung eines festen Körper in einer Flüssigkeit", Math. Ann., Vol.42, (1893), pp.273374.

[53] W. Symes, "Systems of Toda type, inverse spectral problems and representation theory", Invent. Math., Vol.59, (1980), pp.13-53.

[54] M. Toda, "Wave propagation in anharmonic lattices", J. Phys. Soc. of Japan, Vol.23, (1967), pp.501-506.

[55] P. van Moerbeke and D. Mumford, "The spectrum of difference operators and algebraic curves", Acta Math., Vol.143, (1979), pp.93-154.

[56] P. Vanhaecke, Integrable systems in the realm of algebraic geometry, Springer, (2001).

[57] E.T. Whittaker, Analytical Dynamics, Dover, (1944).

[58] S.L. Ziglin, "Splitting of separatrices, branching of solutions and nonexistence of an integral in the dynamics of a solid body", Trans. Moscow Math. Soc., Vol.41, Transl. 1, (1982), pp.283-298.

[59] S.L. Ziglin, "Branching of solutions and the nonexistence of first integrals in Hamiltonian mechanics I, II", Functional Anal. Appl., Vol.16, (1982), pp.181-189. Vol.17, (1983), pp.6-17. 